\title{
A Systematic Review of Randomized Trials of Long-Term Opioid Management for Chronic Non-Cancer Pain
}

Laxmaiah Manchikanti, MD', Hary Ailinani, MD², Dhanalakshmi Koyyalagunta, MD², Sukdeb Datta, $\mathrm{MD}^{4}$, Vijay Singh, $\mathrm{MD}^{5}$, Ike Eriator, $\mathrm{MD}^{6}$, Nalini Sehgal, $\mathrm{MD}^{7}$, Rinoo Shah, $\mathrm{MD}^{8}$, Ramsin Benyamin, MD ${ }^{9}$, Ricardo Vallejo, MD ${ }^{10}$, Bert Fellows, MA ${ }^{11}$, and Paul J. Christo, MD ${ }^{12}$

From: ${ }^{1,11}$ Pain Management Center of Paducah, Paducah, $\mathrm{KY} ;{ }^{2}$ North Shore Long Island Jewish Health System Hyde Park, NY; ${ }^{3} \mathrm{MD}$ Anderson Cancer Center, Dept. Of Anesthesiology \& Pain Medicine, Houston, $\mathrm{TX}_{\text {i }}$ ${ }^{4}$ Vanderbilt University Medical Center, Nashville, TN; ${ }^{5}$ Pain Diagnostics Associates, Niagara, WI: ${ }^{6}$ University of Mississippi Medical Center, Jackson, MS; ${ }^{7}$ University of Wisconsin School of Medicine and Public Health, Madison, $\mathrm{Wl}_{i}$ ${ }^{8}$ Weil-Cornell Medical Center, New York, NY; ${ }^{9,10}$ Millennium Pain Center, Bloomington, IL:

${ }^{12}$ Johns Hopkins University School of Medicine Baltimore, MD

See author affiliations on page

Address Correspondence: Laxmaiah Manchikanti, MD 2831 Lone Oak Road Paducah, Kentucky 42003 E-mail:drlm@thepainmd.com

Disclaimer: There was no external funding in preparation of this manuscript.

Conflict of Interest: Dr. Datta receives research support from Sucampo Pharmaceuticals and an honorarium from Smith and

Nephew

Manuscript received: $11 / 18 / 2010$

Accepted for publication: $01 / 13 / 2011$

Free full manuscript: www.painphysicianjournal.com
Background: Even though opioids have been used for pain for thousands of years, opioid therapy for chronic non-cancer pain is controversial due to concerns regarding the long-term effectiveness and safety, particularly the risk of tolerance, dependance, or abuse. While the debate continues, the use of chronic opioid therapy for chronic non-cancer pain has increased exponentially. Even though evidence is limited, multiple expert panels have concluded that chronic opioid therapy can be effective therapy for carefully selected and monitored patients with chronic non-cancer pain.

Study Design: A systematic review of randomized trials of opioid management for chronic noncancer pain.

Objective: The objective of this systematic review is to evaluate the clinical efficacy of opioids in the treatment of chronic non-cancer pain.

Methods: A comprehensive evaluation of the literature relating to opioids in chronic non-cancer pain was performed. The literature was evaluated according to Cochrane review criteria for randomized controlled trials (RCTs) and Jadad criteria.

A literature search was conducted by using PubMed, EMBASE, Cochrane library, ECRI Institute Library, U.S. Food and Drug Administration (FDA) website, U.S. National Guideline Clearinghouse (NGC), Database of Abstracts of Reviews of Effectiveness (DARE), clinical trials, systematic reviews and cross references from systematic reviews.

The level of evidence was classified as good, fair, or poor based on the quality of evidence developed by the United States Preventive Services Task Force (USPSTF) and used by other systematic reviews and guidelines.

Outcome Measures: Pain relief was the primary outcome measure. Other outcome measures were functional improvement, withdrawals, and adverse effects.

Results: Based on the USPSTF criteria, the indicated level of evidence was fair for Tramadol in managing osteoarthritis. For all the drugs assessed, including Tramadol, for all other conditions, the evidence was poor based on either weak positive evidence, indeterminate evidence, or negative evidence.

Limitations: A paucity of literature, specifically with follow-up beyond 12 weeks for all types of opioids with controlled trials for various chronic non-cancer pain conditions.

Conclusions: This systematic review illustrated fair evidence for Tramadol in managing osteoarthritis with poor evidence for all other drugs and conditions. Thus, recommendations must be based on non-randomized studies.

Key words: Chronic non-cancer pain, opioids, opioid efficacy, opioid effectiveness, significant pain relief, functional improvement, adverse effects, morphine, hydrocodone, hydromorphone, fentanyl, tramadol, buprenorphine, methadone, tapentadol, oxycodone, oxymorphone, systematic reviews, randomized trials

Pain Physician 2011; 14:91-121 
ven though opioids have been used for thousands of years to treat pain, they continue to be one of the most commonly prescribed medications for pain (1-6), and have been well accepted for acute pain, post surgical pain, and palliative care; however, there is debate about whether opioids are appropriate for the treatment of chronic non-cancer pain (1-8). The efficacy of opioids for chronic non-cancer pain has been demonstrated in only short-term trials, including those for neuropathic pain, but the evidence is limited about the efficacy and effectiveness of these agents over the long duration of treatment typical for chronic non-cancer pain $(1-4,7,8)$.

Chronic pain has been defined by the American Society of Interventional Pain Physicians (ASIPP) as, "pain that persists 6 months after an injury and beyond the usual course of an acute disease or a reasonable time for a comparable injury to heal, that is associated with chronic pathologic processes that cause continuous or intermittent pain for months or years, that may continue in the presence or absence of demonstrable pathologies; may not be amenable to routine pain control methods; and healing may never occur" $(9,10)$. Persistent pain interfering with daily activities is common; however, chronic persistent pain is separate from chronic pain syndrome which has been defined as a complex condition with physical, psychological, emotional, and social components. The prevalence of chronic pain in the adult population ranges from $2 \%$ to $40 \%$ with a median point prevalence of $15 \%$ (9-12). Further, age related prevalence of persistent pain appears to be much more common in the elderly associated with functional limitations and difficulty in performing daily life activities (11-14).

Several published guidelines and consensus statements recommend the judicious use of opioids in appropriately selected patients with chronic non-cancer pain who have not responded to other treatments and analgesic medications (1-4,7,8,15-17). Also, multiple systematic reviews have been conducted evaluating the efficacy, effectiveness, side effects, abuse and diversion, and other factors $(7,8,18-31)$. However, concrete evidence of the effectiveness and safety of opioids in chronic pain has not been demonstrated. The foundation of the argument for the use of opioids is the unique analgesic efficacy of opioids, based on surveys, case series, occasional open-label follow-up studies, as well as some randomized controlled trials (RCTs) and epidemiological studies. Recent guidelines by Chou and Huffman (8) and Noble et al (7) yielded useful guidance. Noble et al (7) concluded that many patients discontinue long-term opioid therapy due to adverse events or insufficient pain relief; however, weak evidence suggests that patients who were able to continue opioids long-term experience clinically significant pain relief. The findings regarding quality of life or functional improvement were inconclusive. They also cautioned that the evidence supporting these conclusions is weak, and longer-term studies are needed to identify the patients who are more likely to benefit from treatment. Chou and Huffman (8) concluded that chronic opioid therapy can be an effective therapy for carefully selected and monitored patients with chronic non-cancer pain. They also pointed out that opioids are also associated with potentially serious harms, including opioid-related adverse effects and outcomes related to the abuse potential of opioids. Nevertheless, both guidelines recommended opioids in the face of weak evidence.

The purpose of this systematic review is to summarize the evidence pertaining to the efficacy of longterm opioid therapy for chronic non-cancer pain.

\subsection{Methods}

The methodology utilized here follows the systematic review process derived from evidence-based systematic review and meta-analysis of randomized trials (32-39), Consolidated Standards of Reporting Trials (CONSORT) guidelines for the conduct of randomized trials $(40,41)$, Cochrane guidelines (7), Chou and Huffman (8) guidelines, and Quality of Reporting of Metaanalyses (QUOROM) (35) and Preferred Reporting Items for Systematic Reviews and Meta-analyses (PRISMA) statement (36) for conduct of systematic reviews and meta-analyses.

\subsection{Criteria for Consideration of the Studies}

\subsubsection{Types of Studies}

- Randomized controlled trials (RCTs).

\subsubsection{Types of Participants}

- Adults aged at least 18 years with pain due to any cause other than cancer lasting for at least 3 months prior to trial enrollment.

- Previous non-opioid pharmacotherapy must have failed before beginning opioids.

\subsubsection{Types of Interventions}

- Any opioid administered either orally or topically.

- Any dose for at least 12 weeks. 


\subsection{Types of Outcome Measures}

- Minimum of 12 weeks of follow-up.

- Pain relief.

- Average change in pain scores.

- Proportion of patients with at least $50 \%$ pain relief.

- Health-related quality of life and function.

\subsection{Adverse Events or Side Effects}

- Discontinuation from study due to adverse events.

- Discontinuation from study due to insufficient pain relief.

\subsection{Search Methods for Identification of Studies} Searches were performed from the following sources:

1. PubMed from 1966

www.ncbi.nlm.nih.gov/sites/entrez?db=pubmed

2. EMBASE from 1980

www.embase.com/

3. Cochrane Library

www.thecochranelibrary.com/view/0/index.html

4. ECRI Institute Library

www.ecri.org/Pages/default.aspx

5. U.S. Food and Drug Administration (FDA) website from 1977

www.usda.gov/wps/portal/usda/usdahome

6. U.S. National Guideline Clearinghouse (NGC) from 1998

www.guideline.gov/

7. Previous systematic reviews and cross references

8. Database of Abstracts of Reviews of Effectiveness (DARE)

www.crd.york.ac.uk/crdweb/Home.aspx?DB=DARE

9. Clinical Trials

clinicaltrials.gov/

Search period included from 1966 to September 2010.

\subsection{Search Strategy}

The search terminology included RCTs, chronic non-cancer pain, all types of chronic pain (nociceptive, neuropathic, and visceral; and low back, thoracic, neck, musculoskeletal, rheumatic, localized, generalized, chest, headache, joint pain, arthritis, psychogenic pain), all types of opioids (morphine, codeine, oxymorphone, methadone, oxycodone, hydrocodone, hydromorphone, oxymorphone, dihydrocodeine, tramadol, fentanyl, levorphanol, buprenorphine, propoxyphene, meperidine, tapentadol, and pentazocine).

At least 2 of the review authors independently, in an unblinded standardized manner, performed each search. Accuracy was confirmed by a statistician. All searches were combined to obtain a unified search strategy. Any disagreements between reviewers were resolved by a third author and consensus.

\subsection{Data Collection and Analysis}

\subsubsection{Selection of Studies}

- Two review authors screened the abstracts, in an unblinded standardized manner, of all identified studies against the inclusion criteria.

- They then retrieved all possibly relevant articles in full text for comprehensive assessment of internal validity, quality, and satisfaction of inclusion criteria.

\subsubsection{Assessment of Methodologic Quality}

Two review authors independently assessed, in an unblinded standardized manner, the internal validity of all the studies.

The methodologic quality assessment was performed in a manner to avoid any discrepancies which were evaluated by a third reviewer and consensus was reached.

Methodologic quality assessment criteria are described in Tables 1 and $2(37,38)$.

\subsubsection{Data Extraction and Management}

Two review authors independently, in an unblinded standardized manner, extracted the data from the included studies. Disagreements were resolved by discussion between the 2 review authors; if no agreement could be reached, it was planned a third author would decide.

\subsubsection{Assessment of Heterogeneity}

Whenever meta-analysis was conducted, the Isquared (I2) statistic was used to identify heterogeneity (42). Combined results with $12>50 \%$ were considered substantially heterogenous.

We divided the evidence base by mode of drug administration, either topical or oral, to reduce clinical heterogeneity.

\subsubsection{Measurement of Treatment Effect and Data Synthesis (Meta-Analysis)}

Data were summarized using meta-analysis when at least 5 studies per type of opioid administration addressed chronic non-cancer pain (e.g., tra- 
Table 1. Criteria list for methodological quality assessment*.

\begin{tabular}{|c|c|c|}
\hline Criteria & Operationalization of Criteria & Score \\
\hline $\begin{array}{l}\text { A. Was the method of randomization } \\
\text { adequate? }\end{array}$ & $\begin{array}{l}\text { A random (unpredictable) assignment sequence. An example of adequate meth- } \\
\text { ods is a computer generated random number table and use of sealed opaque en- } \\
\text { velopes. Methods of allocation using DOB, date of admission, hospital numbers, } \\
\text { or alternation should not be regarded as appropriate. }\end{array}$ & $\begin{array}{l}\text { Yes/No/ } \\
\text { Don't Know }\end{array}$ \\
\hline B. Was the treatment allocation concealed? & $\begin{array}{l}\text { Assignment generated by an independent person not responsible for determin- } \\
\text { ing the eligibility of the patients. This person has no information about the } \\
\text { persons included in the trial and has no influence on the assignment sequence } \\
\text { or on the decision about eligibility of the patient. }\end{array}$ & $\begin{array}{l}\text { Yes/No/ } \\
\text { Don't Know }\end{array}$ \\
\hline $\begin{array}{l}\text { C. Were the groups similar at baseline } \\
\text { regarding the most important prognostic } \\
\text { factors? } \\
\text { "Yes", if similar: } \\
\text { - Age \& gender } \\
\text { - Description of type of pain } \\
\text { - Intensity, duration or severity of pain }\end{array}$ & $\begin{array}{l}\text { In order to receive a "yes," groups have to be similar in baseline regarding demo- } \\
\text { graphic factors, duration or severity of complaints, percentage of patients with } \\
\text { neurologic symptoms, and value of main outcome measure(s). }\end{array}$ & $\begin{array}{l}\text { Yes/No/ } \\
\text { Don't Know }\end{array}$ \\
\hline $\begin{array}{l}\text { D. Was the patient blinded to the } \\
\text { intervention? }\end{array}$ & \multirow{3}{*}{$\begin{array}{l}\text { The reviewer determines if enough information about the blinding is given in } \\
\text { order to score a "yes": Use the author's statement on blinding, unless there is a } \\
\text { differing statement/reason not to (no need for explicit information on blinding). } \\
\text { If a study notes it is double-blind, code "yes" for patient, care provider and out- } \\
\text { come assessor (unless it is clear that one of these is not blinded) }\end{array}$} & \multirow{3}{*}{$\begin{array}{l}\text { Yes/No/ } \\
\text { Don't Know }\end{array}$} \\
\hline $\begin{array}{l}\text { E. Was the care provider blinded to the } \\
\text { intervention? }\end{array}$ & & \\
\hline $\begin{array}{l}\text { F. Was the outcome assessor blinded to the } \\
\text { intervention? }\end{array}$ & & \\
\hline $\begin{array}{l}\text { G. Were cointerventions avoided or } \\
\text { similar? }\end{array}$ & $\begin{array}{l}\text { Cointerventions should either be avoided in the trial design or similar between } \\
\text { the index and control groups. Code "yes" if there is a statement about co-inter- } \\
\text { vention medications being used or not used, e.g.: rescue analgesics not allowed } \\
\text { or note about which rescue analgesics were permitted or if rescue analgesics are } \\
\text { outcomes. }\end{array}$ & $\begin{array}{l}\text { Yes/No/ } \\
\text { Don't Know }\end{array}$ \\
\hline $\begin{array}{l}\text { H. Was the compliance acceptable in all } \\
\text { groups? }\end{array}$ & $\begin{array}{l}\text { The reviewer determines if the compliance to the interventions is acceptable, } \\
\text { based on the reported intensity, duration, number and frequency of sessions for } \\
\text { both the index intervention and control intervention(s). Code "yes" if protocol } \\
\text { violations are reported or if actual compliance data is reported. }\end{array}$ & $\begin{array}{l}\text { Yes/No/ } \\
\text { Don't Know }\end{array}$ \\
\hline $\begin{array}{l}\text { I. Was the drop-out rate described and } \\
\text { acceptable? } \\
\leq 15 \% \text { drop out rate is acceptable. }\end{array}$ & $\begin{array}{l}\text { The number of participants who are included in the study but did not complete } \\
\text { the observation period or were not included in the analysis must be described } \\
\text { and reasons given. If the percentage of withdrawals and drop-outs does not } \\
\text { exceed } 15 \% \text { and does not lead to substantial bias, a "yes" is scored. }\end{array}$ & $\begin{array}{l}\text { Yes/No/ } \\
\text { Don't Know }\end{array}$ \\
\hline $\begin{array}{l}\text { J. Was the timing of the outcome } \\
\text { assessment in all groups similar? }\end{array}$ & $\begin{array}{l}\text { Timing of outcome assessment should be identical for all intervention groups } \\
\text { and for all important outcome assessments. }\end{array}$ & $\begin{array}{l}\text { Yes/No/ } \\
\text { Don't Know }\end{array}$ \\
\hline $\begin{array}{l}\text { K. Did the analysis include an intention-to- } \\
\text { treat analysis? } \\
\text { "Yes" if less than } 5 \% \text { of no-treatment excluded. }\end{array}$ & $\begin{array}{l}\text { All randomized patients are reported/analyzed in the group they were allocated } \\
\text { to by randomization for the most important moments of effect measurement } \\
\text { (minus missing values) irrespective of noncompliance and cointerventions. }\end{array}$ & $\begin{array}{l}\text { Yes/No/ } \\
\text { Don't Know }\end{array}$ \\
\hline
\end{tabular}

* Table adapted from methods developed by the Cochrane Back Review Group (van Tulder, Furlan, Bombardier, Bouter, and Editorial Board of the Cochrane Collaboration Back Review Group) Spine (Phila Pa 1976) 2003; 28:1290-1299 (37). 
Table 2. Jadad quality rating for primary studies*.

\begin{tabular}{|c|c|c|c|}
\hline Criteria & Scoring & Operationalization of Criteria & $\begin{array}{l}\text { Criteria } \\
\text { Score }\end{array}$ \\
\hline $\begin{array}{l}\text { Randomization: Was the study } \\
\text { described as randomized (use of } \\
\text { words such as randomly, random, } \\
\text { and randomization)? }\end{array}$ & $\begin{array}{l}\text { Yes }=1 \\
\text { No }=0\end{array}$ & $\begin{array}{l}\text { Add } 1 \text { point if: Method to generate the sequence of randomization was } \\
\text { described and was appropriate (e.g. computer-generated, table of random } \\
\text { numbers, etc.) and adequate method used for allocation concealment (e.g., } \\
\text { centralized randomization or opaque, sealed envelopes) } \\
\text { Subtract } 1 \text { point if: Method of randomization described and inappropriate } \\
\text { (e.g.,alternating patients, different hospital, etc.) }\end{array}$ & $0-2$ \\
\hline $\begin{array}{c}\text { Blinding: Was the study described } \\
\text { as double-blind? }\end{array}$ & $\begin{array}{l}\text { Yes }=1 \\
\text { No }=0\end{array}$ & $\begin{array}{l}\text { Add } 1 \text { point if: Method of double blinding described and appropriate (identical } \\
\text { placebo, active placebo, term "double-dummy" used) } \\
\text { Subtract } 1 \text { point if: Method of double blinding described and inappropriate } \\
\text { (comparison of tablets that are not identical-appearing) }\end{array}$ & $0-2$ \\
\hline $\begin{array}{l}\text { Withdrawals and drop-outs: Was } \\
\text { there a description of withdrawals } \\
\text { and dropouts? }\end{array}$ & $\begin{array}{l}\text { Yes }=1 \\
\text { No }=0\end{array}$ & Only 0 or 1 possible. & 0 or 1 \\
\hline \multicolumn{4}{|c|}{ OVERALL SCORE } \\
\hline
\end{tabular}

* Jadad AR et al. Assessing the quality of reports of randomized clinical trials: Is blinding necessary? Controlled Clin Trials 1996; 17:1-12 (38).

madol - 5 studies meeting inclusion criteria evaluating individual conditions of chronic pain), low back pain or osteoarthritis. Qualitative (the direction of a treatment effect) and quantitative (the magnitude of a treatment effect) conclusions were evaluated. Random-effects meta-analyses to pool data were also used (39).

The minimum amount of change in pain score to be clinically meaningful has been described as a 2point change on a scale of 0 to 10 (or 20 percentage points), based on findings in trials studying general chronic pain (43), chronic musculoskeletal pain (44), and chronic low back pain $(32-34,45,46)$, which have been commonly utilized. However, recent descriptions of clinically meaningful improvement have been described as significant improvement, either with pain relief or functional status as $50 \%$ (47-50). Consequently, for this analysis, we have utilized clinically meaningful pain relief of at least a 4-point change on an 11 -point scale of 0 to 10 , or $50 \%$ pain relief from the baseline as clinically significant.

\subsubsection{Integration of Heterogeneity}

The evidence was assessed separately by mode of administration, either oral or transdermal, by the drug administered (i.e., morphine, oxymorphone, etc.), and by the predominant pain condition treated (i.e., low back pain, osteoarthritis, etc.). The meta- analysis was performed only if there were at least 5 studies meeting inclusion criteria available for each variable.

Statistical heterogeneity was explored using univariate meta-regression (51).

\subsubsection{Software Used for Assessment}

The data were analyzed using SPSS (9.0) statistical software (SPSS Inc., Chicago, IL), Microsoft Access 2003, and Microsoft Excel 2003 (Microsoft Corporation, Redmond, WA) (52).

Meta-analyses were done with Comprehensive Meta-Analysis software version 2.0 for Windows (Biostat Inc., Englewood, NJ) (53).

\subsection{Summary Measures}

Summary measures included $50 \%$ or more reduction of pain in at least $40 \%$ of the patients, or at least 4 points decrease in pain scores and relative risk of adverse events including side effects and abuse patterns.

\subsection{Analysis of Evidence}

Analysis of evidence was performed based on United States Preventative Services Task Force (USPSTF) criteria (Table 3) (54), which have been utilized by others (8). 
Table 3. Method for grading the overall strength of the evidence for an intervention.

\begin{tabular}{|c|l||}
\hline Grade & \multicolumn{1}{|c|}{ Definition } \\
\hline Good & $\begin{array}{l}\text { Evidence includes consistent results from well-designed, well-conducted } \\
\text { studies in representative populations that directly assess effects on health outcomes (at least 2 consistent, higher-qual- } \\
\text { ity RCTs or studies of diagnostic test accuracy). }\end{array}$ \\
\hline Fair & $\begin{array}{l}\text { Evidence is sufficient to determine effects on health outcomes, but the strength of the evidence is limited by the } \\
\text { number, quality, size, or consistency of included studies; generalizability to routine practice; or indirect nature of the } \\
\text { evidence on health outcomes (at least one higher-quality trial or study of diagnostic test accuracy of sufficient sample } \\
\text { size; } 2 \text { or more higher-quality trials or studies of diagnostic test accuracy with some inconsistency; at least } 2 \text { consistent, } \\
\text { lower-quality trials or studies of diagnostic test accuracy, or multiple consistent observational studies with no signifi- } \\
\text { cant methodological flaws). }\end{array}$ \\
\hline Poor & $\begin{array}{l}\text { Evidence is insufficient to assess effects on health outcomes because of limited number or power of studies, large and } \\
\text { unexplained inconsistency between higher-quality trials, important flaws in trial design or conduct, gaps in the chain } \\
\text { of evidence, or lack of information on important health outcomes. }\end{array}$ \\
\hline
\end{tabular}

Source: Chou R, Huffman L. Use of Chronic Opioid Therapy in Chronic Noncancer Pain: Evidence Review. American Pain Society; Glenview, IL: 2009 (8). Adapted from methods developed by U.S. Preventive Services Task Force (54).

\subsection{Results}

\subsection{Study Selection}

Figure 1 shows a flow diagram of the study selection as recommended by Preferred Reporting Items for Systematic Reviews and Meta-Analyses (PRISMA) (36).

\subsection{Inclusion Criteria}

Of the 111 randomized trials identified (55-165), Table 4 illustrates the list of excluded studies, the majority of them being for short-term follow-up, whereas some other studies were excluded due to secondary analysis, evaluation of breakthrough pain, postsurgical pain, or drug levels.

Table 5 illustrates assessment of the 23 trials for inclusion criteria. Twenty-one studies met inclusion criteria (143-151,153-160,162-165). Thus, 2 of the 23 studies were excluded from the methodologic quality assessment $(152,161)$.

\subsection{Methodologic Quality Assessment}

A methodologic quality assessment of the studies meeting inclusion criteria was carried out utilizing Cochrane review criteria and Jadad criteria as shown in Tables 6 and 7. Studies achieving Cochrane scores of 9 or higher and Jadad criteria of at least 4 were considered as high quality, 6 to 8 of Cochrane and Jadad criteria of at least 3 were considered as moderate quality, whereas 5 to 6 of Cochrane and at least 2 of Jadad were considered as low quality. Studies scoring less than 5 on Cochrane review and/or less than 2 on Jadad score were excluded.

Nine studies were considered as high quality with Cochrane scores of 9 or higher of 11 and Jadad scores of at least 4 of $5(147-149,151,153,156,158,162,164)$. Six studies were considered as of moderate quality with 6 to 8 of 11 Cochrane criteria and at least 3 of 5 Jadad criteria $(143,145,150,155,157,163)$, whereas 5 studies were considered low quality based on Cochrane review criteria scores of 5 to $6(144,146,154,159,165)$, and at least 2 of Jadad criteria. One study (160) scored 3 of 11 of Cochrane criteria; thus, was excluded from further analysis.

On the included condition-specific studies, 8 studies evaluated low back pain $(144,148,151,154,156,163$ $165), 4$ studies evaluated chronic pain $(146,149,159,162)$, 8 studies evaluated osteoarthritis $(143,145,147,150,155$, $157,158,165)$, and one study evaluated diabetic neuropathy (153).

Of the 8 studies evaluating low back pain, 3 were considered as low quality $(144,154,165)$, one was considered as moderate quality (163), and 4 were considered as high quality $(148,151,156,164)$.

Of the 4 studies evaluating chronic pain, 2 were considered as low quality $(146,159)$ and 2 were considered as high quality $(149,162)$.

Of the 8 studies evaluating osteoarthritis, one study was of low quality (165), one study was of moderate quality (155), and 6 studies were of high quality $(143,145,147,150,157,158)$. 
10,336 records identified through database searching

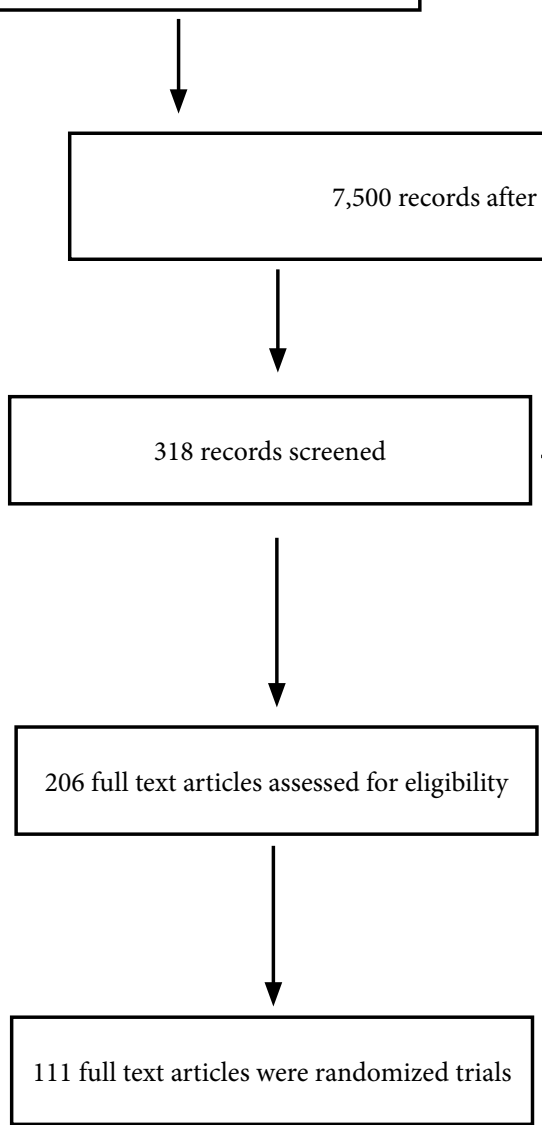

48 additional records identified through other sources

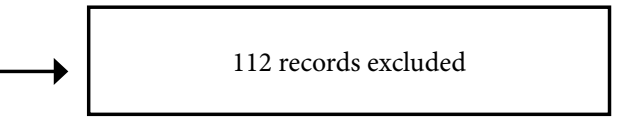

Fig. 1. Flow diagram of literature search.

The only study evaluating diabetic neuropathy (153) was rated as high quality.

\subsection{Meta-Analysis}

All the studies were evaluated for inclusion of meta-analysis.

Oxycodone was evaluated in 4 trials for its effectiveness in low back pain $(148,154,164,165), 3$ trials in chronic pain $(146,159,162), 3$ trials in osteoarthritis $(143,157,165)$, and one trial in diabetic neuropathy (153).

Tramadol was evaluated for its use in osteoarthritis in 5 trials $(145,147,150,155,158)$, of which 2 studied osteoarthritis of the knee $(145,158)$ and one studied osteoarthritis of the knee and hip (155) and one for management of low back pain (163).

Morphine was evaluated for managing chronic pain in 2 trials $(149,159)$ and low back pain in 2 trials $(144,154)$.

Oxymorphone was studied in 2 trials for low back pain $(151,156)$. Fentanyl was evaluated for low back pain in one trial (144).

Hydromorphone was evaluated for chronic pain in one study (146).

Tapentadol was evaluated for osteoarthritis in 2 trials $(143,165)$ and in 2 trials for low back pain 
Pain Physician: March/April 2011; 14:91-121

Table 4. List of excluded studies.

\begin{tabular}{|c|c|c|c|}
\hline \multirow{2}{*}{ Manuscript Author(S) } & \multirow{2}{*}{ Drugs Studied } & \multicolumn{2}{|c|}{ Reason for Exclusion } \\
\hline & & Follow-up Period & Other Reasons \\
\hline Adler et al 2002 (55) & Tramadol & 3 weeks & \\
\hline Aqua et al 2007 (56) & Oxymorphone & 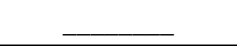 & Postoperative pain \\
\hline Aurilio et al 2009 (57) & Buprenorphine & 4 weeks & \\
\hline Beaulieu et al 2007 (58) & Tramadol & 4 weeks & \\
\hline Beaulieu et al 2008 (59) & Tramadol & 8 weeks & \\
\hline Bodalia et al $2003(60)$ & Tramadol & 2 weeks & \\
\hline Caldwell et al 1999 (61) & Oxycodone & 5 weeks & \\
\hline Caldwell et al 2002 (62) & Morphine & 4 weeks & \\
\hline Chang et al 2009 (63) & Hydromorphone & & Intravenous postoperative \\
\hline Chindalore et al 2005 (64) & Oxycodone & 3 weeks & \\
\hline Cowan et al 2005 (65) & Morphine & & Abstinence \\
\hline Daniels et al 2009 (66) & Tapentadol/oxycodone & + & Postoperative \\
\hline Daniels et al 2009 (67) & Tapentadol/oxycodone & 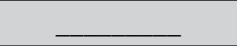 & Postoperative \\
\hline Etropolski et al $2010(68)$ & Tapentadol & 4 weeks & Dose conversion \\
\hline Frank et al 2008 (69) & Dihydrocodeine & 2 weeks & \\
\hline Gatti et al 2009 (70) & Morphine & 5 weeks & Breakthrough pain \\
\hline Gilron et al 2005 (71) & Morphine & 5 weeks & \\
\hline Gimbel et al 2003 (72) & Oxycodone & 6 weeks & \\
\hline Gordon et al 2010 (73) & Buprenorphine & 6 weeks & \\
\hline Gordon et al $2010(74)$ & Buprenorphine & 8 weeks & \\
\hline Gould et al 2009 (75) & Oxymorphone & 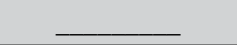 & Secondary analysis \\
\hline Grosset et al $2005(76)$ & Hydromorphone & 1 week & \\
\hline Hale et al 1997 (77) & Codeine & 1 week & \\
\hline Hale et al 2005 (78) & Oxymorphone & 3 weeks & \\
\hline Hale et al $1999(79)$ & Oxycodone & 2 weeks & \\
\hline Hale et al 2007 (80) & Oxycodone & 6 weeks & \\
\hline Hamann \& Sloan 2007 (81) & Morphine & 1 week & Role of oral naltrexone in intrathecal morphine \\
\hline Harati et al $2000(82)$ & Tramadol & 6 weeks & \\
\hline Harke et al 2001 (83) & Morphine & 8 days & \\
\hline Hartrick et al 2009 (84) & Tapentadol/oxycodone & 2 weeks & \\
\hline Huse et al 2001 (85) & Morphine & 4 weeks & \\
\hline James et al $2010(86)$ & Buprenorphine & 7 weeks & \\
\hline Jensen \& Ginsberg 1994 (87) & Tramadol & 2 weeks & \\
\hline Kalso et al 2007 (88) & Transdermal Fentanyl and Morphine & $\bar{L}$ & Secondary analysis \\
\hline Katz et al 2010 (89) & Morphine & 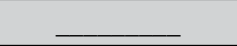 & Pharmacokinetics \\
\hline Khoromi et al 2007 (90) & Morphine & 9 weeks & \\
\hline Kivitz et al 2006 (91) & Oxymorphone & 2 weeks & \\
\hline Kleinert et al 2008 (92) & Tapentadol & $<1$ day & Post-surgical pain \\
\hline Landau et al 2007 (93) & Buprenorphine & 5 weeks & \\
\hline Lange et al 2010 (94) & Tapentadol \& oxycodone & Pooled analysis & \\
\hline Langford et al 2006 (95) & Fentanyl & 6 weeks & \\
\hline Likar et al 2007 (96) & Buprenorphine & 2 weeks & \\
\hline Litkowski et al 2005 (97) & Oxycodone & 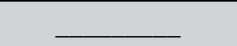 & Post op dental pain \\
\hline Ma et al $2008(98)$ & Oxycodone & 4 weeks & \\
\hline
\end{tabular}


Systematic Review of Opioids in Chronic Pain

Table 4 (cont.). List of excluded studies.

\begin{tabular}{|c|c|c|c|}
\hline \multirow{2}{*}{ Manuscript Author(S) } & \multirow{2}{*}{ Drugs Studied } & \multicolumn{2}{|c|}{ Reason for Exclusion } \\
\hline & & Follow-up Period & Other Reasons \\
\hline Malonne et al 2004 (99) & Tramadol & 2 weeks & \\
\hline Malonne et al 2005 (100) & Tramadol & 4 weeks & \\
\hline Matsumoto et al 2005 (101) & Oxymorphone & 4 weeks & \\
\hline Max et al 1988 (102) & Codeine & 6 hours & \\
\hline Mcllwain and Ahdieh 2005 (103) & Oxymorphone & 3 weeks & \\
\hline Morley et al 2003 (104) & Methadone & 2 day & \\
\hline Moulin et al 1996 (105) & Morphine & 9 weeks & \\
\hline Mullican et al 2001 (106) & Tramadol & 4 weeks & \\
\hline Munera et al 2010 (107) & Buprenorphine & 5 weeks & \\
\hline Nicholson et al 2006 (108) & Morphine & 2 weeks & \\
\hline Niemann et al 2000 (109) & Morphine vs Fentanyl & 4 weeks & \\
\hline Norrbrink \& Lundeberg 2009 (110) & Tramadol & 4 weeks & \\
\hline Palangio et al 2002 (111) & Hydrocodone vs. oxycodone & 1 week & \\
\hline Parris et al 1998 (112) & Oxycodone & 1 week & \\
\hline Paulson et al 2005 (113) & Alvimopan & 3 weeks & \\
\hline Perrot et al 2006 (114) & Tramadol & $<2$ weeks & \\
\hline Petrone et al 1999 (115) & Tramadol & 4 weeks & \\
\hline Portenoy et al 2007 (116) & Fentanyl & + & Breakthrough pain \\
\hline Raber et al 1999 (117) & Tramadol & 2 weeks & \\
\hline Raja et al 2002 (118) & Morphine and methadone & 8 weeks & \\
\hline Ralphs et al 1994 (119) & Opiate reductions & 4 weeks & \\
\hline Rauck et al 2006 (120) & Morphine/oxycodone & 4 weeks & \\
\hline Roth et al $2000(121)$ & Oxycodone & 5 weeks & \\
\hline Rowbotham et al 2003 (122) & Levorphanol & 8 weeks & \\
\hline Ruoff 1999 (123) & Tramadol & 2 weeks & \\
\hline Ruoff et al 2003 (124) & Tramadol & 2 weeks & \\
\hline Salzman et al 1999 (125) & Oxycodone & 3 weeks & \\
\hline Sandner-Kiesling et al 2010 (126) & Oxycodone \& naloxone & Pooled analysis & \\
\hline Simpson et al 2007 (127) & Fentanyl & 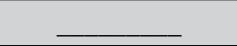 & Breakthrough pain \\
\hline Sindrup et al 1999 (128) & Tramadol & 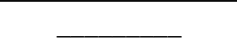 & Drug levels \\
\hline Sindrup et al 1999 (129) & Tramadol & 4 weeks & \\
\hline Sorge \& Stadler 1997 (130) & Tramadol & 3 weeks & \\
\hline Sorge and Sittl 2004 (131) & Buprenorphine & $<1$ week & \\
\hline Stegmann et al 2008 (132) & Tramadol & 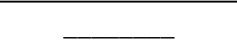 & Post operative pain \\
\hline Tessaro et al 2010 (133) & Oxycodone & 4 weeks & \\
\hline Thorne et al 2008 (134) & Tramadol & 8 weeks & \\
\hline Vorsanger et al 2007 (135) & Tramadol & Post hoc analysis & \\
\hline Vorsanger et al 2010 (136) & Tapentadol, oxycodone & Post hoc analysis & \\
\hline Wallace et al 2007 (137) & Hydromorphone & 6 weeks & \\
\hline Watson \& Babul 1998 (138) & Oxycodone & 4 weeks & \\
\hline Watson et al 2003 (139) & Oxycodone & 4 weeks & \\
\hline Webster et al 2008 (140) & Alvimopan & 6 weeks & \\
\hline Wilder-Smith 2001 (141) & Tramadol/dihydrocodeine & 4 weeks & \\
\hline Zautra \& Smith 2005 (142) & Oxycodone & 2 weeks & \\
\hline
\end{tabular}




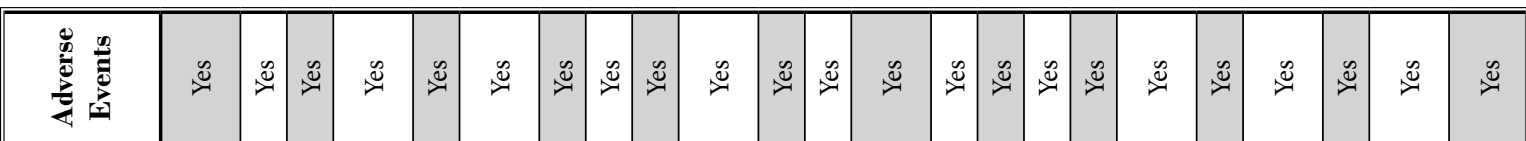

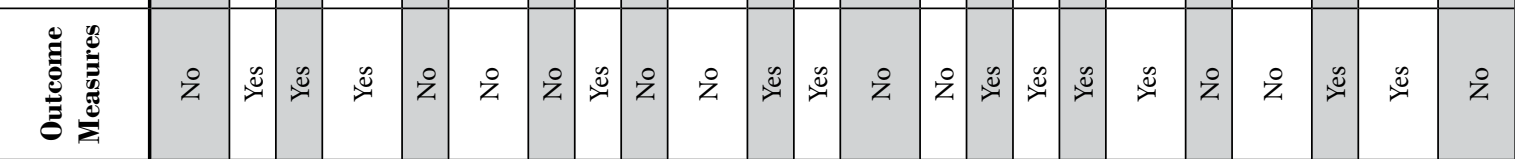

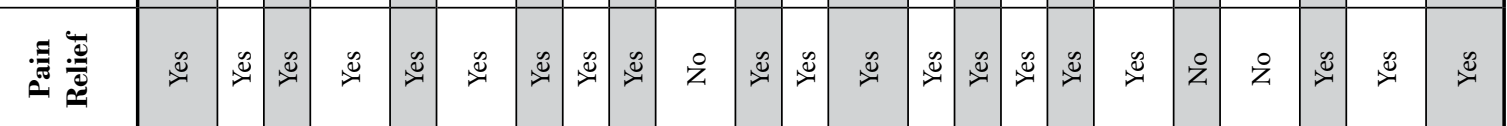

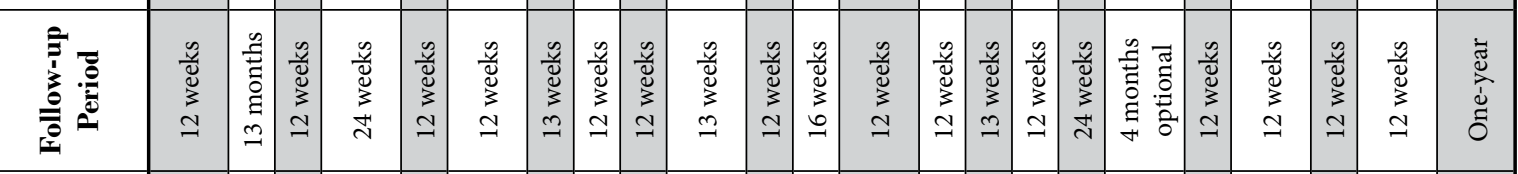

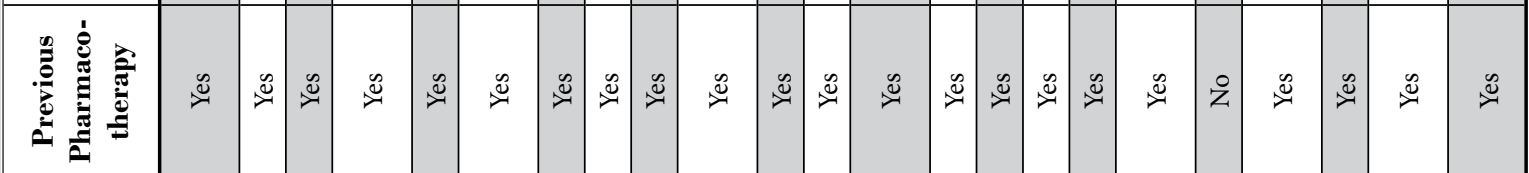

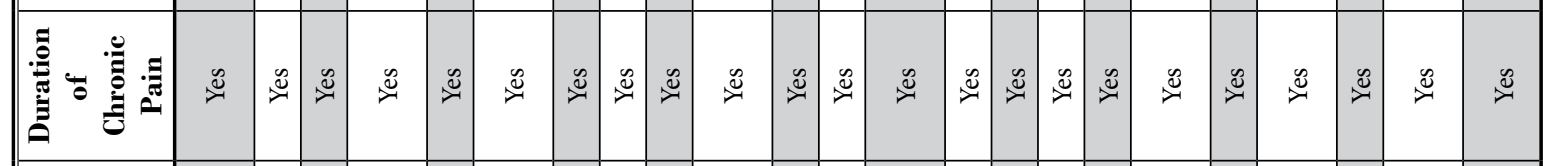

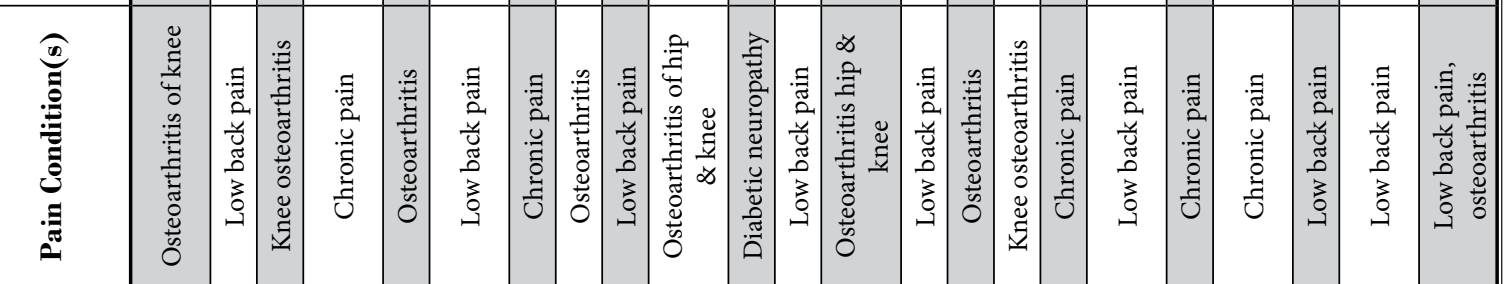

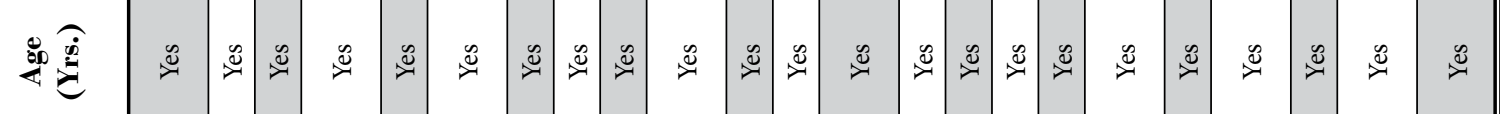

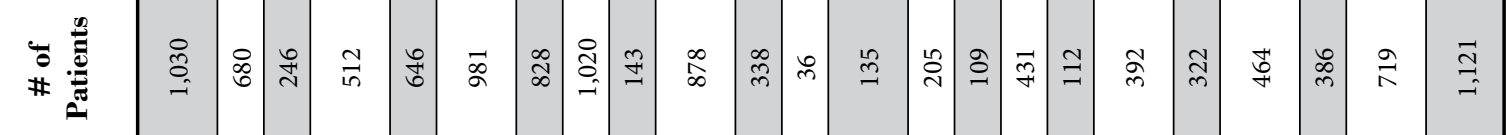

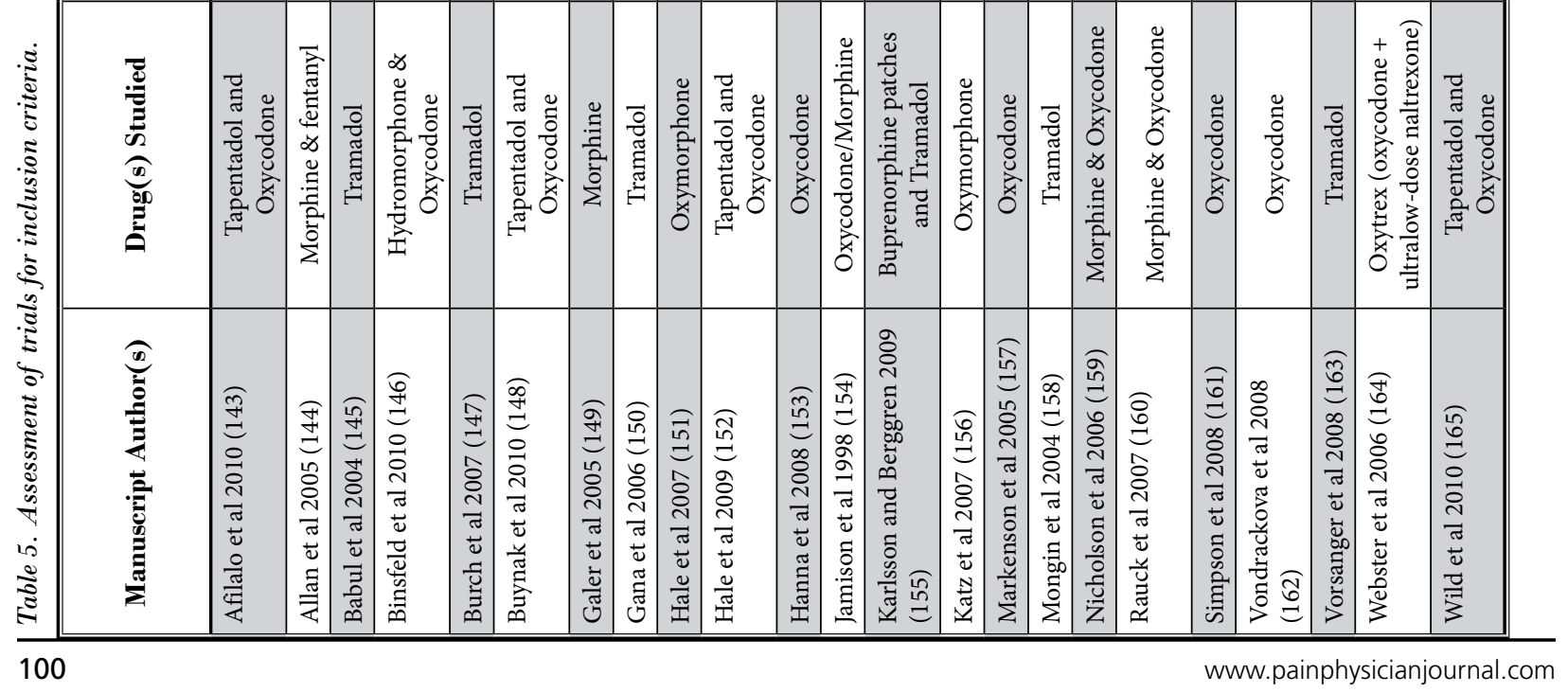




\begin{tabular}{|c|c|c|c|c|c|c|c|c|c|c|c|c|c|c|c|c|c|c|c|c|c|}
\hline 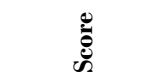 & $\underset{\infty}{\vec{\nabla}}$ & $\vec{\nabla}$ & $\underset{\infty}{\vec{\infty}}$ & $\vec{\nabla}$ & $\vec{\Xi}$ & $\vec{\Xi}$ & 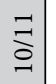 & $\underset{\infty}{\vec{\sigma}}$ & $\bar{\Xi}$ & $\vec{\xi}$ & $\vec{F}$ & $\underset{N}{\Xi}$ & $\underset{\Xi}{\stackrel{\Xi}{9}}$ & $\vec{\infty}$ & $\underset{\Xi}{\Xi}$ & $\vec{F}$ & $\underset{m}{\vec{m}}$ & $\underset{\Xi}{\Xi}$ & $\vec{\infty}$ & $\vec{\sigma}$ & in \\
\hline 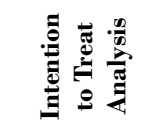 & $\stackrel{\circ}{Z}$ & 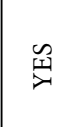 & $\stackrel{\circ}{Z}$ & 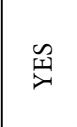 & 吕 & そ) & પ્ર & 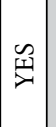 & 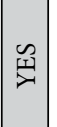 & 㜽 & $\stackrel{\circ}{z}$ & 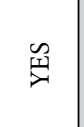 & 出 & $\stackrel{\circ}{z}$ & 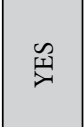 & $\stackrel{\circ}{z}$ & $\begin{array}{l}0 \\
z\end{array}$ & 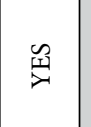 & 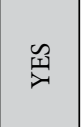 & ’ & 䜣 \\
\hline 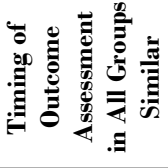 & 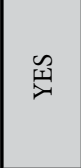 & 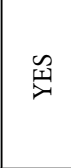 & 墨 & 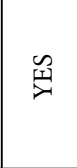 & 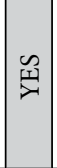 & $\stackrel{\infty}{ \pm ્ \lambda}$ & 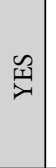 & 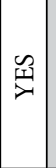 & 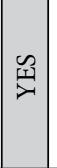 & 亗 & 亗 & 亗 & 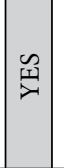 & $\stackrel{\leftrightarrow}{\Theta ్}$ & 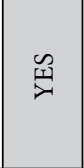 & $\stackrel{\infty}{\mathscr{N}}$ & 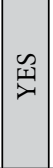 & 亗 & 㜽 & 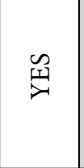 & 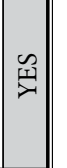 \\
\hline 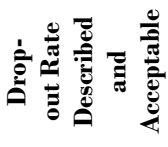 & そ & 尺े & $\stackrel{0}{Z}$ & そ) & $\stackrel{\circ}{z}$ & そ) & $\begin{array}{l}O \\
Z\end{array}$ & $\begin{array}{l}o \\
z\end{array} \mid$ & $\begin{array}{l}\wp \\
z\end{array}$ & そ) & 亗 & 亗 & ̊̊ & $\stackrel{\bigcirc}{Z}$ & 亗 & ̊ి & $\stackrel{0}{Z}$ & $\stackrel{\mathscr{I}}{\underline{\lambda}}$ & $\stackrel{\circ}{z}$ & そ) & $\stackrel{\circ}{z}$ \\
\hline 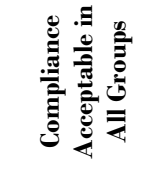 & ○) & $\stackrel{\circ}{z}$ & $\stackrel{\circ}{Z}$ & $\stackrel{\circ}{Z}$ & $\stackrel{一}{Z}$ & 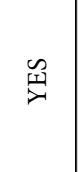 & 笎 & 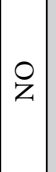 & $\begin{array}{l}\wp \\
z\end{array}$ & そ) & 㜽 & 亗 & 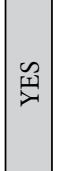 & $\stackrel{\bigcirc}{Z}$ & ભ્ર & ભ્ર & $\begin{array}{l}0 \\
z\end{array}$ & 㜽 & ஜ̊ & 巴્રે & $\stackrel{\circ}{z}$ \\
\hline 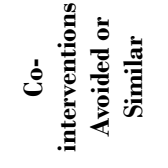 & ભ્રે & ભ્રે & 点 & 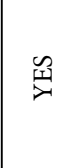 & 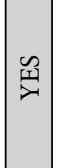 & 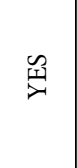 & 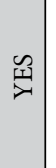 & 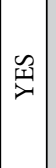 & 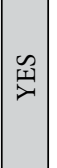 & 至 & 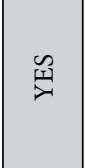 & પ્રે & ભ્રે & 吕 & Щ્ર & ભ્ર & 亗 & 呈 & 㜽 & 亗 & 饥 \\
\hline 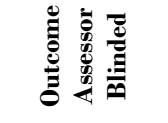 & 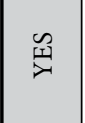 & $\stackrel{\circ}{z}$ & 版 & $\stackrel{\circ}{z}$ & 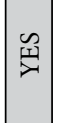 & 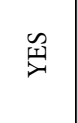 & 至 & 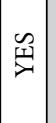 & 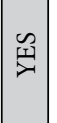 & 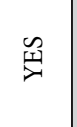 & $\stackrel{\circ}{Z}$ & $\stackrel{\circ}{Z}$ & 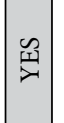 & 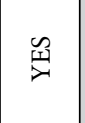 & 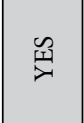 & $\stackrel{O}{Z}$ & $\begin{array}{l}0 \\
z\end{array}$ & 出 & હ્ર & ભ્રે & $\stackrel{\circ}{Z}$ \\
\hline 吾 & 圤 & そ) & 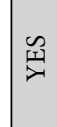 & $\stackrel{\circ}{\gtrless}$ & 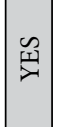 & 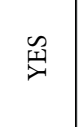 & 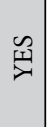 & 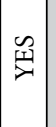 & 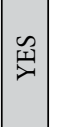 & 亗 & $\stackrel{\circ}{Z}$ & そ) & జ્ર & $\stackrel{\sim}{\underline{\lambda}}$ & 㜽 & $\stackrel{\circ}{\text { Z }}$ & $\stackrel{0}{Z}$ & 㜽 & ભ્ર & 苂 & $\stackrel{\circ}{z}$ \\
\hline 兽 & 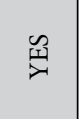 & 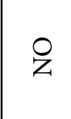 & 至 & $\stackrel{\circ}{z}$ & $\begin{array}{l}\tilde{y} \\
\tilde{\lambda}\end{array}$ & 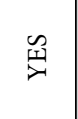 & ભ્રે & 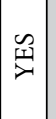 & $\begin{array}{l}\tilde{y} \\
\text { I્રે }\end{array}$ & 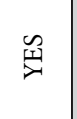 & $\stackrel{\wp}{Z}$ & そ) & પ્ર્ત & ભ્ર & ભ્રે & そ) & $\begin{array}{l}0 \\
z\end{array}$ & $\stackrel{\infty}{્}$ & પ્ર્ત & $\stackrel{\infty}{\underbrace{2}}$ & $\stackrel{\circ}{z}$ \\
\hline 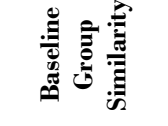 & 纤 & 㜽 & 出 & 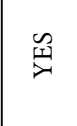 & 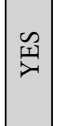 & 亗 & પ્ર & $\stackrel{\circ}{z}$ & 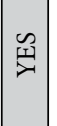 & 亗 & 尺) & 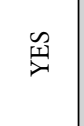 & 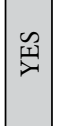 & 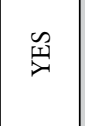 & 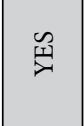 & $\stackrel{\bigcirc}{Z}$ & $\stackrel{0}{Z}$ & $\stackrel{\circ}{z}$ & $\stackrel{\circ}{\text { Z }}$ & 出 & 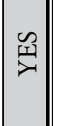 \\
\hline 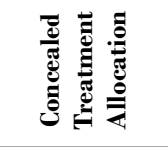 & 巫 & $\stackrel{̊}{z}$ & 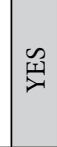 & $\stackrel{\circ}{z}$ & 亗 & 㜽 & 忞 & 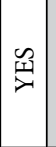 & 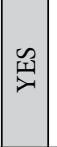 & 出 & $\stackrel{\circ}{Z}$ & そ) & 出 & 巴્ર & ભ્રે & ભ્રે & $\stackrel{0}{Z}$ & 亗 & 㜽 & 亗 & $\stackrel{\bigcirc}{Z}$ \\
\hline 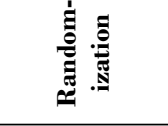 & 纤 & 㜽 & 至 & 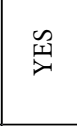 & ભ્રે & 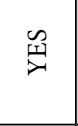 & પ્ર & 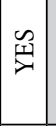 & 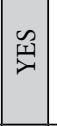 & 亗 & લ્ર & 島 & 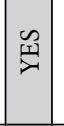 & 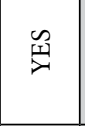 & પ્ર & 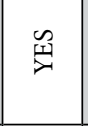 & 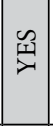 & 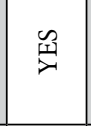 & ભ્ર & 出 & 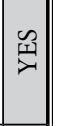 \\
\hline 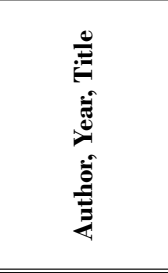 & 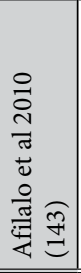 & 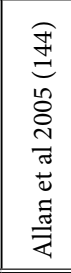 & 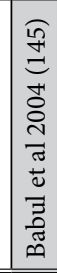 & 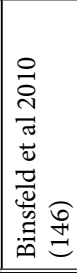 & 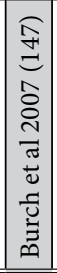 & 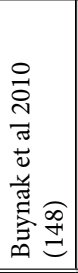 & 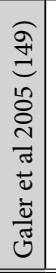 & 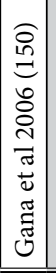 & 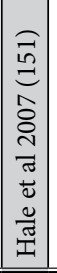 & 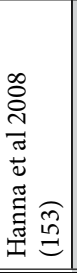 & 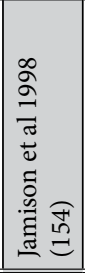 & 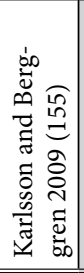 & 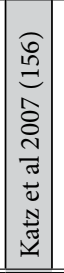 & 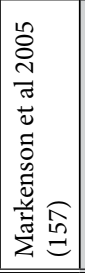 & 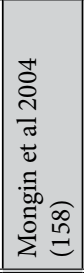 & 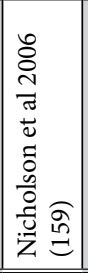 & 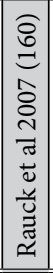 & 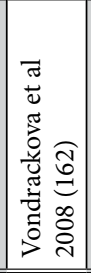 & 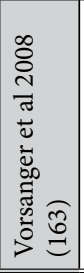 & 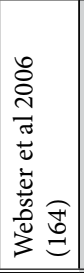 & 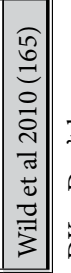 \\
\hline
\end{tabular}


$(148,165)$.

There was one trial which evaluated Buprenorphine for osteoarthritis (155).

None of the drugs met inclusion criteria for metaanalysis; thus, no meta-analysis was performed.

\subsection{Study Characteristics}

Table 8 illustrates the study characteristics of the included studies evaluating the efficacy of opioids.

\subsection{Methodologic Quality Assessment for Bias}

Methodologic quality assessment data utilizing Cochrane review criteria is illustrated in Table 6 . This table shows adequate data with regards to adequacy of randomization, concealment allocation, and blinding of patients. Twenty-one of 23 studies were assessed for quality assessment. Of these, one trial (160) evaluating morphine and oxycodone in low back pain was excluded due to low quality scores.

Blinding of patients, health care providers, data collectors, and outcome assessors were also evaluated utilizing Cochrane review criteria as shown in Table 6, which were present in 15 of the 20 trials evaluated. However, 16 of 20 studies were deficient with regards to dropouts, loss to follow-up, and other reasons. They are considered the major disadvantage of all the trials evaluated for this systematic review.

\subsection{Analysis of Evidence}

\subsubsection{Tramadol}

Tramadol was assessed in 6 randomized trials (145, $147,150,155,158,163)$. The effectiveness of tramadol in managing chronic low back pain was evaluated in one study (163), 2 studies evaluated osteoarthritis of the knee $(145,158)$, one studied osteoarthritis of the knee and hip (155), and the other 2 studied osteoarthritis of various joints $(147,150)$. None of the studies provided data in terms of $50 \%$ pain relief. Thus, the criteria of reduction of at least 4 points or $40 \%$ in the pain scores was considered as significant.

Table 7. Methodologic quality assessment of randomized trials utilizing Jadad scoring criteria.

\begin{tabular}{|c|c|c|c|c|}
\hline Author, Year, Title & Randomization & Blinding & $\begin{array}{l}\text { Reporting of } \\
\text { Withdrawals }\end{array}$ & Score \\
\hline Afilalo et al 2010 (143) & 2 & 2 & 1 & $5 / 5$ \\
\hline Allan et al 2005 (144) & 2 & 0 & 1 & $3 / 5$ \\
\hline Babul et al 2004 (145) & 2 & 2 & 1 & $5 / 5$ \\
\hline Binsfeld et al 2010 (146) & 2 & 0 & 1 & $3 / 5$ \\
\hline Burch et al 2007 (147) & 2 & 2 & 1 & $5 / 5$ \\
\hline Buynak et al 2010 (148) & 2 & 2 & 1 & $5 / 5$ \\
\hline Galer et al 2005 (149) & 2 & 2 & 1 & $5 / 5$ \\
\hline Gana et al $2006(150)$ & 1 & 2 & 1 & $4 / 5$ \\
\hline Hale et al 2007 (151) & 2 & 2 & 0 & $4 / 5$ \\
\hline Hanna et al 2008 (153) & 2 & 2 & 1 & $5 / 5$ \\
\hline Jamison et al 1998 (154) & 1 & 0 & 1 & $2 / 5$ \\
\hline Karlsson and Berggren 2009 (155) & 2 & 0 & 1 & $3 / 5$ \\
\hline Katz et al 2007 (156) & 2 & 2 & 1 & $5 / 5$ \\
\hline Markenson et al 2005 (157) & 2 & 2 & 1 & $5 / 5$ \\
\hline Mongin et al 2004 (158) & 1 & 2 & 1 & $4 / 5$ \\
\hline Nicholson et al 2006 (159) & 1 & 0 & 1 & $2 / 5$ \\
\hline Rauck et al 2007 (160) & 1 & 0 & 1 & $2 / 5$ \\
\hline Vondrackova et al 2008 (162) & 2 & 2 & 1 & $5 / 5$ \\
\hline Vorsanger et al 2008 (163) & 2 & 2 & 1 & $5 / 5$ \\
\hline Webster et al 2006 (164) & 1 & 2 & 1 & $4 / 5$ \\
\hline Wild et al 2010 (165) & 2 & 0 & 1 & $3 / 5$ \\
\hline
\end{tabular}




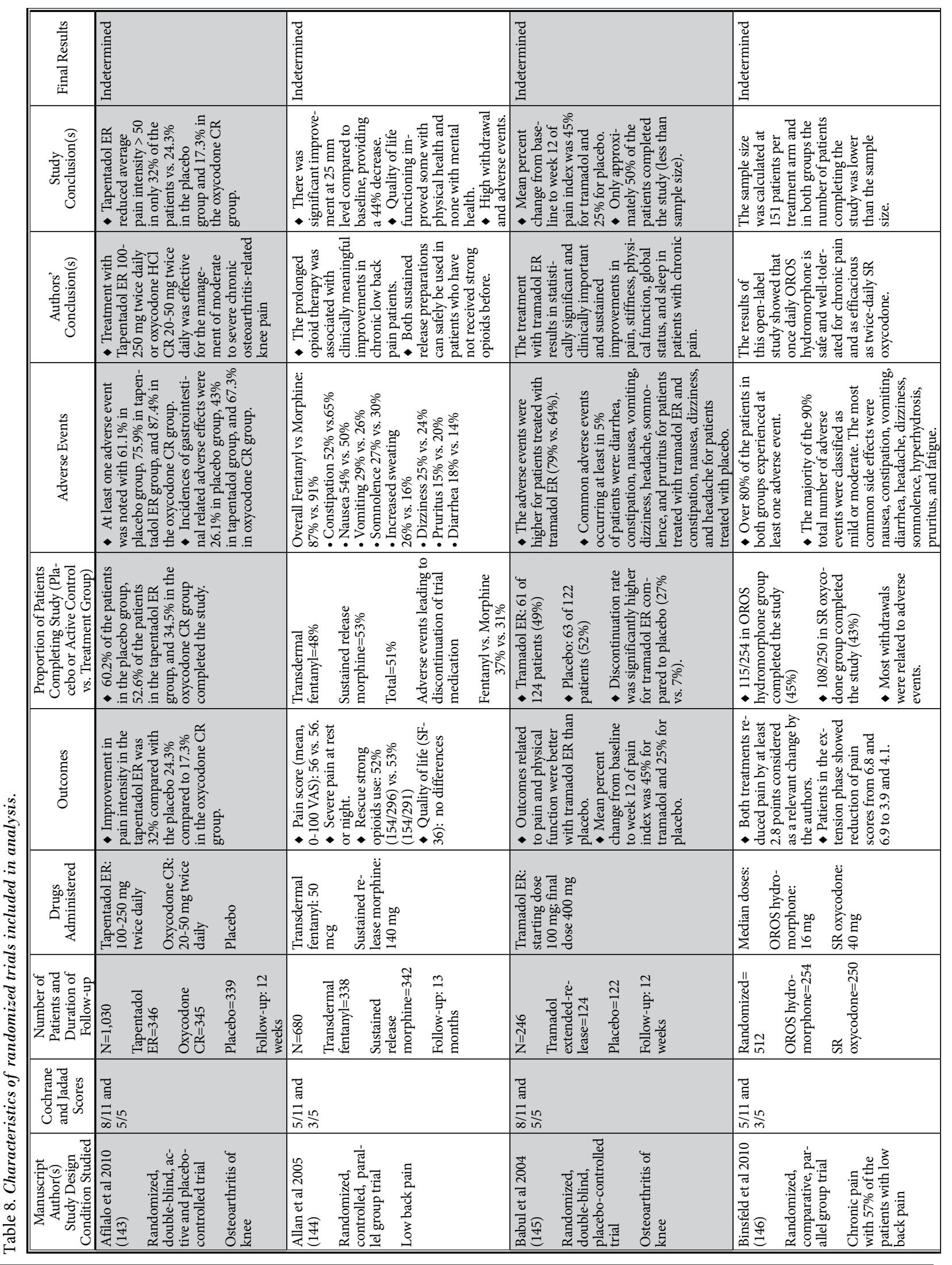

www.painphysicianjournal.com 


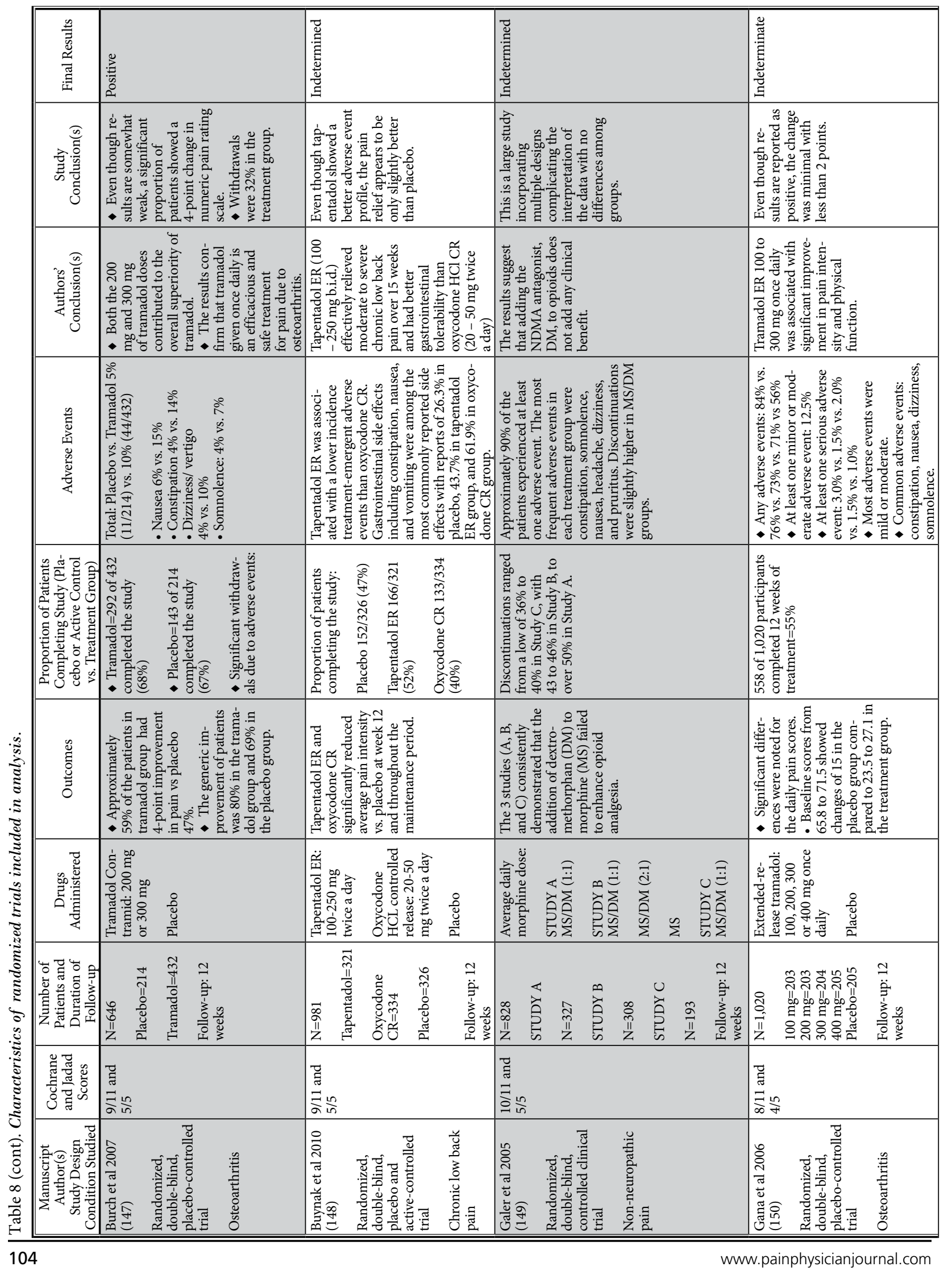




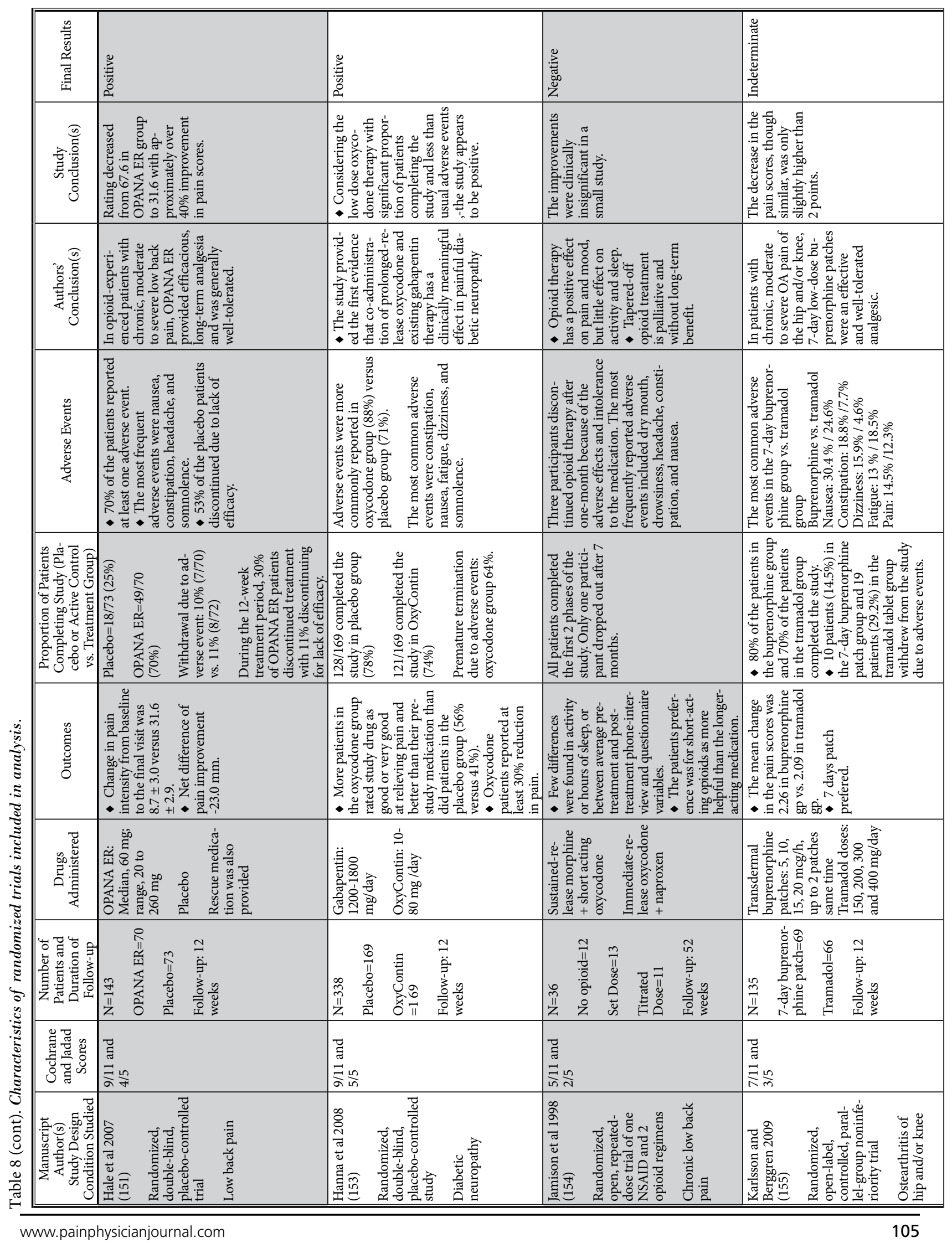




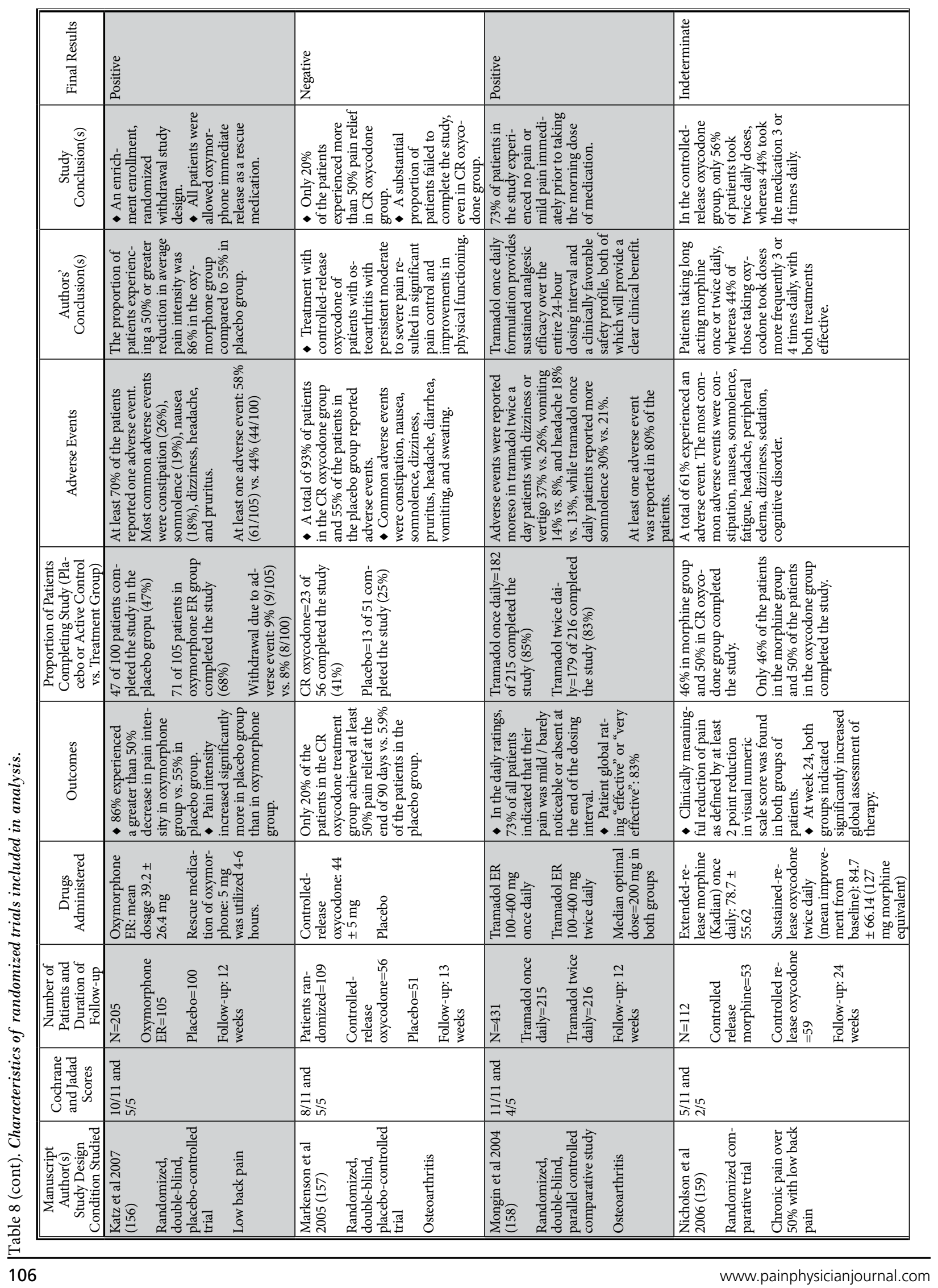




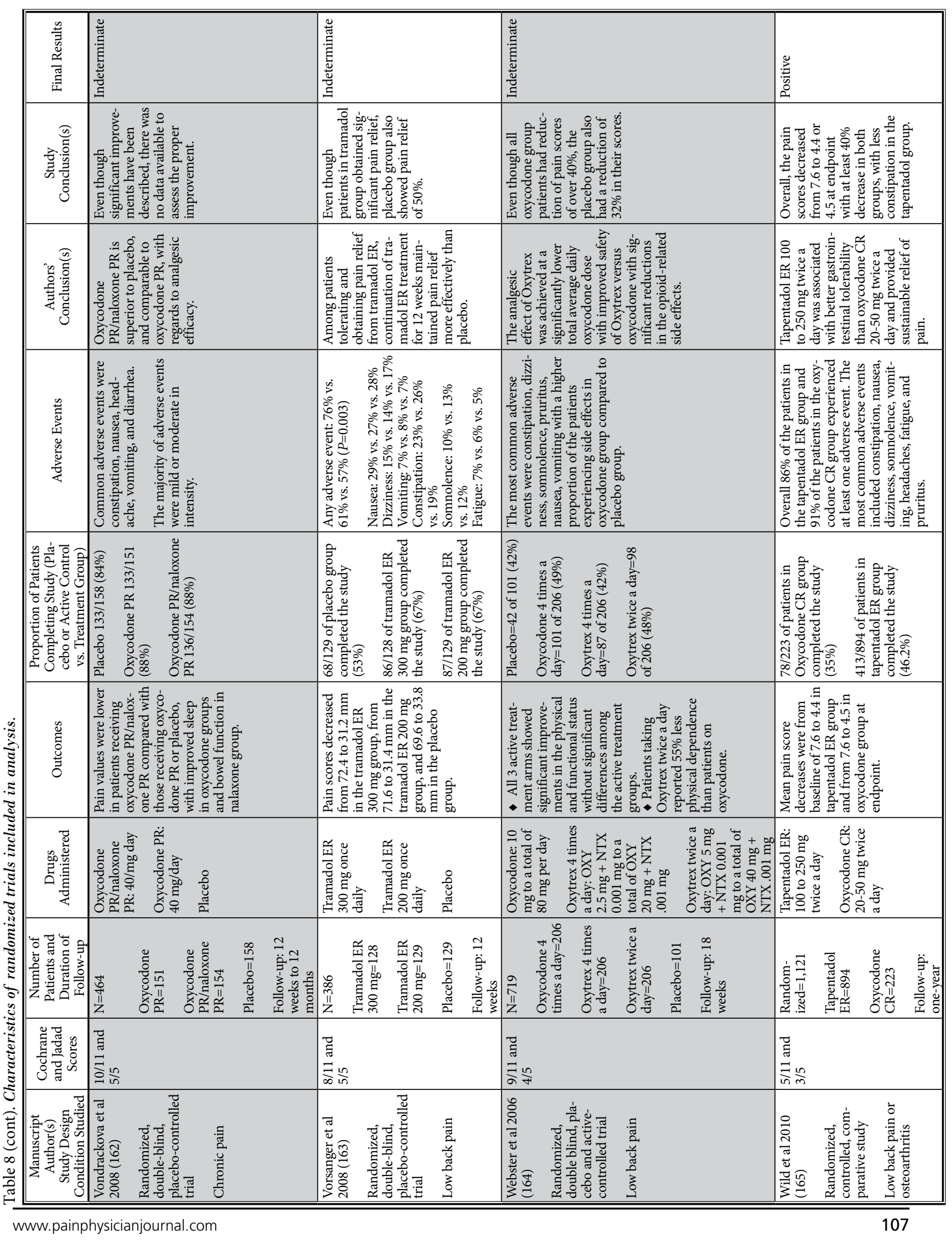


Of the 3 studies evaluating arthritis of multiple joints $(147,150,155)$, only one was positive (147) and 2 studies were indeterminate $(150,155)$. Between the 2 studies evaluating osteoarthritis of the knee $(145,158)$, one study was positive (158) and the second study was indeterminate (145). The single study evaluating effectiveness in low back pain (163) was indeterminate.

\subsubsection{Strength of Evidence}

Based on grading for overall strength of evidence for intervention as illustrated in Table 3, the evidence for tramadol in managing various chronic painful conditions is variable from fair to poor. For osteoarthritis of multiple joints, of the 2 placebo controlled trials $(147,150)$ with high methodologic quality, one study was shown to be positive (147) and the second one was shown to be indeterminate (150). The third study was a parallel group comparison study (155) with moderate methodologic quality; it was indeterminate, with the summary of evidence leading to an assessment of fair for osteoarthritis of multiple joints.

In managing osteoarthritis of the knee, one placebo-controlled trial with high methodologic quality was indeterminate (145), a second comparative trial with high methodologic quality (158) showed positive results, and a third trial showed indeterminate results in a parallel group study (155) with the summary of evidence as fair.

In managing low back pain, there was only one placebo-controlled study (163) with high methodologic quality criteria, which was shown to be indeterminate, with the summary of evidence as poor.

\subsubsection{Oxycodone}

Oxycodone was evaluated for its role in managing chronic pain of various types in 10 studies (143, $146,148,153,154,157,159,162,164,165)$. Of these, researchers evaluated effectiveness for low back pain in 4 studies $(148,154,164,165)$, for chronic non-cancer pain in 3 studies $(146,159,162)$, for osteoarthritis in 3 studies $(143,157,165)$, and in one trial the role of oxycodone in patients receiving gabapentin in diabetic neuropathy (153). Of the 10 studies, 2 of them included $50 \%$ pain relief as the criterion standards, whereas the remaining used various types of criteria.

Of the 10 reports provided, 2 studies, a high quality, placebo-controlled trial (153), and a low quality comparative trial (165), provided positive evidence;
6 trials, 4 of which were placebo-controlled trials with high methodologic quality $(143,148,162,164)$, and 2 comparative trials with low methodologic quality $(146,159)$, provided indeterminate evidence; and 2 studies, a placebo controlled study with high methodologic quality (157) and an open study with low methodologic quality (154) provided negative evidence.

\subsubsection{Strength of Evidence}

Based on grading for overall strength of evidence for an intervention as illustrated in Table 3, for low back pain, the evidence was poor with a low quality open label study showing negative results (154) and 2 placebo-controlled high quality studies $(148,164)$ showing indeterminate results.

For chronic pain, one placebo-controlled, high quality study (162) and 2 comparative trials with low methodologic quality assessment $(146,159)$, all showed indeterminate results. Consequently, the evidence was poor in managing chronic pain.

For management of osteoarthritis, of the 2 high quality placebo-controlled trials $(143,157)$, one was indeterminate (143) and the second one was negative (157), whereas one low quality comparative trial (165) showed positive results with an overall conclusion of poor evidence.

For diabetic neuropathy, only one placebo controlled trial of high quality (153) showed weak positive evidence, with overall poor evidence.

\subsubsection{Morphine}

Four randomized trials were identified evaluating the role of morphine in managing chronic pain of various types $(144,149,154,159)$. Of these, 2 low quality trials evaluated low back pain $(144,154)$ and 2 studies, one high quality (149) and one low quality (159), evaluated chronic pain. None of the trials were placebo-controlled.

\subsubsection{Strength of Evidence}

Based on grading for overall strength of evidence for an intervention as illustrated in Table 3, the evidence for morphine in managing chronic low back pain or chronic non-cancer pain was poor.

Two non-placebo controlled trials evaluating low back pain, one parallel group (144) and one comparative trial (154), with low quality of methodology, showed either indeterminate (144) or negative evidence (154). For chronic pain, 2 comparative trials, one high quality 
(149) and the second one low quality (159), showed indeterminate evidence.

\subsubsection{Oxymorphone}

Two trials evaluated efficacy and safety of oxymorphone in patients with chronic low back pain; one study recruited opioid naïve patents (156) and the other study enlisted opioid experienced patients (151). Both were randomized placebo-controlled, enriched enrollment trials and were graded as high quality studies. However a significant number of participants in both studies dropped out; as a result at the final assessment the total number of subjects in each study was less than the number calculated for powering the study. In the study by Hale (151), 60 patients per treatment group were needed to provide $90 \%$ power at a $5 \%$ significance level, while the participants that completed the study were $49 / 70$ in the treatment arm and $18 / 73$ in the placebo arm. In the Katz study (156), a smaller effect size (0.45) was anticipated and it was estimated that 80 patients per treatment group were needed to provide $80 \%$ power at $5 \%$ significance. The number of participants completing the study was $71 / 105$ in the treatment group and $47 / 100$ in the placebo group. Even though both studies demonstrated that compared to patients in the placebo group, a higher percentage of patients in the treatment group reported clinically significant pain relief, the significance of these findings is questionable because of the high drop out rate and failure to meet the number needed to power the study.

\subsubsection{Strength of Evidence}

Based on available evidence $(151,156)$, i.e., only 2 studies of insufficient power, there is not enough evidence to assess the efficacy of oxymorphone on outcomes in patients with chronic low back pain. Hence, we conclude the overall strength of evidence as illustrated in Table 3, is poor.

\subsubsection{Tapentadol}

Three studies evaluated the role of tapentadol in managing osteoarthritis and low back pain $(143,148,165)$. Of the 3 studies, one low quality study evaluated both osteoarthritis and low back pain (165) with positive results, one high quality study evaluated ostearthritis of the knee (143), and one high quality study that evaluated low back pain only (148) showed indeterminate results.

\subsubsection{Strength of Evidence}

Based on the grading of overall strength of evidence as illustrated in Table 3, evidence is poor for tapentadol in managing osteoarthritis and chronic low back pain, with one low quality study being positive with weak evidence (165) and 2 high quality studies being indeterminate $(143,148)$.

\subsubsection{Fentanyl}

Fentanyl was assessed in only one low quality, randomized, parallel group trial evaluating low back pain (144). Results of this study were indeterminate.

\subsubsection{Strength of Evidence}

Based on grading for overall strength of evidence as illustrated in Table 3, the evidence is poor for fentanyl in managing low back pain with one low quality, parallel group, randomized trial, with indeterminate evidence (144).

\subsubsection{Hydromorphone}

One low quality, randomized, comparative trial evaluated hydromorphone comparing it with oxycodone in managing chronic pain (146).

\subsubsection{Strength of Evidence}

Based on grading for overall strength of evidence as illustrated in Table 3, the evidence was poor for hydromorphone for managing chronic pain with one low quality comparative trial showing indeterminate evidence (146).

\subsubsection{Buprenorphine}

One moderate quality, open-label, parallel group randomized trial evaluated transdermal buprenorphine with tramadol in managing osteoarthritis of the hip and knee (155) with indeterminate results.

\subsubsection{Strength of Evidence}

Based on grading for overall strength of evidence as illustrated in Table 3, the evidence was poor for transdermal buprenorphine for managing ostearthritis based upon a single moderate quality, randomized, comparative trial (155).

\subsection{Discussion}

In this systematic review, the efficacy of opioids (transdermal fentanyl and buprenorphine, oral morphine, tramadol, oxycodone, oxymorphone, tapentadol, and hydromorphone) was evaluated in patients 
with multiple pain conditions including chronic pain, low back pain, osteoarthritis, and diabetic neuropathy. The results showed fair evidence for administration of tramadol in osteoarthritis of multiple joints, and knee osteoarthritis. However, for all other agents, including tramadol, in all conditions, the evidence was very weak or negative leading to the conclusion of poor evidence.

This systematic review evaluated only randomized trials with a minimum 12-week follow-up, meeting the inclusion criteria, as well as methodologic quality assessment criteria. Thus, the results of the efficacy evaluation might be somewhat different than previous systematic reviews and guideline syntheses, leading to differences in conclusions.

Tramadol was assessed in 6 randomized trials $(145,147,150,155,158,163)$ and of these, 4 were placebo controlled $(145,147,150,163)$, one was comparative (158) and one a parallel group trial (155). Among the studies evaluating the role of tramadol in osteoarthritis of multiple joints, of the 2 placebo controlled trials $(147,150)$, one was positive and the second one was indeterminate. The third parallel-group trial (155) was also indeterminate. All the authors concluded that tramadol provided statistically and clinically significant improvement in pain relief. These studies also concluded that tramadol provided in divided doses or as once daily extended release was well tolerated and effective; however, the results showed borderline results and significant withdrawals. Burch et al (147) showed weak results, but a significant proportion of patients showed a 4-point change in Numeric Rating Scale (NRS) with withdrawals of $32 \%$ in the treatment group. This was the only study with a weak but positive conclusion by the authors of the systematic review. The other 2 studies $(150,155)$, one placebo controlled and the second one a parallel group, showed results which were indeterminate. Gana et al (150) showed a positive change with less than 2 points, whereas Karlsson and Berggren (155) also showed a change of approximately 2 points.

In evaluation of the role of tramadol in osteoarthritis of the knee, 2 studies $(145,158)$ were included with one placebo controlled trial (145) and the second one a comparative trial (158). Even though the authors concluded in both studies that tramadol was an effective modality for osteoarthritis of the knee, the mean percentage change from baseline was $45 \%$ for tramadol and $25 \%$ for placebo (145), whereas in the second study by Mongin et al (158), $73 \%$ of the patients in the study experienced no pain or mild pain immedi- ately prior to taking the morning dose of medication and had transient and non-serious side effects. Thus, the comparative evaluation (158) was considered as a positive trial and the placebo controlled trial (145) was indeterminate.

The sole study of low back pain (163) was a placebo controlled trial with indeterminate results. The authors concluded that among patients tolerating and obtaining pain relief from tramadol, it provided good pain relief compared to the placebo; however, patients in the tramadol group as well as the placebo group obtained significant relief. The differences between the reduction of pain with placebo versus tramadol were not substantially higher.

Consequently, even though tramadol is presented to show fair evidence, it is weak.

Oxycodone, one of the most commonly used drugs, was evaluated for its role in managing chronic pain of various types in 10 studies $(143,146,148,153,154,157$, $159,162,164,165)$. However, only one high quality placebo-controlled trial (153) and a low quality comparative trial (165) provided positive evidence. In evaluation of oxycodone, there were 4 studies evaluating low back pain $(148,154,164,165)$. One comparative study with low methodologic quality was positive (165). Two placebo controlled trials $(148,164)$, both with high quality, were indeterminate, one open-label study with low quality (154) was negative.

In evaluation of chronic pain, all 3 trials met inclusion criteria $(146,159,162)$. One placebo-controlled with high methodologic quality criteria (162), and 2 comparative trials with low methodologic quality assessment criteria $(146,159)$, were indeterminate.

For osteoarthritis, of the 3 studies $(143,157,165)$, 2 were placebo-controlled and high quality $(143,157)$ and one was a comparative trial with low methodologic quality (165) judged to be positive, whereas of the 2 high quality placebo-controlled trials, one was indeterminate (143) and the second one was negative (157). The one single trial evaluating diabetic neuropathy, which was placebo-controlled with high methodologic quality (153), was positive.

Consequently, based on the above synthesis of evidence, on balance most authors concluded that either oxycodone was effective or safer than other drugs compared, even though the effectiveness illustrated was not substantially higher than other groups, with tapentadol and tramadol. The side effects were lower in those drugs than oxycodone. The effect on physical functioning, mood, and activity was also low. There was 
a significant proportion of withdrawals in patients receiving oxycodone. Adding naltrexone has been touted as advantageous, even though the evidence is indeterminate. Consequently, despite its extensive use and the large number of studies, the evidence was poor for low back and chronic pain, osteoarthritis, and diabetic neuropathy, based on either negative, indeterminate, or very weak positive evidence.

It was surprising that morphine was evaluated in only 4 trials that met the inclusion criteria $(144,149,154,159)$ and only one of them was of high quality (149). Of the 2 low quality studies evaluating low back pain, one was negative (154) and the second one was indeterminate (144), whereas for chronic pain, both studies were indeterminate, with one low quality comparative trial (159) and the second one a high quality comparative trial (149).

Consequently, the administration of morphine for multiple conditions showed a lack of significant evidence.

Oxymorphone, an agent which is not commonly used, was evaluated in 2 studies $(151,156)$ for low back pain yielding poor evidence. One placebo controlled trial, methodologically of high quality, showed positive results (151); however, these results were weak. An improvement was seen of approximately $40 \%$ in pain scores; however, rescue medication was provided to the majority of the patients. The second study (156) reported $50 \%$ or greater reduction in average pain intensity in $86 \%$ of the patients receiving oxymorphone. However, $55 \%$ of the patients in the placebo group also obtained $50 \%$ pain relief. Both studies used an enrichment enrollment protocol with a high rate of responders; also, break through oxymorphone immediate release was available for all patients. These studies were inadequately powered due to high drop out rates and failure to meet the number of participants needed to power the study.

Tapentadol, a relatively new drug, and most commonly used in acute pain, has been studied for managing chronic pain of osteoarthritis and low back in 3 randomized trials $(143,148,165)$. Of the 3 studies evaluating tapentadol, 2 scored high on methodologic quality assessment $(143,148)$, whereas the third study was comparative with low methodologic quality assessment (165). Both placebo controlled trials $(143,148)$, one evaluating osteoarthritis (143) and one evaluating low back pain (148), even though methodologically of high quality, provided indeterminate evidence with only $32 \%$ of the patients receiving greater than $50 \%$ pain relief for osteoarthritis (143). One comparative evaluation with low methodologic quality showed positive results (165). Thus, even though tapentadol at the present time has poor evidence, it appears that this new drug may have potential, similar to effects as other opioids, and with fewer side effects.

Allan et al (144) compared transdermal fentanyl with sustained release morphine. They concluded that transdermal fentanyl and sustained release morphine both provided excellent pain relief, but morphine was associated with more constipation. Even though results appear to be positive, both groups showed high withdrawal rates and high adverse events with significant improvement at a $25 \mathrm{~mm}$ level compared to baseline. The study also showed that there was quality of life function improvement with physical health, but none with mental health. Consequently, though commonly used, transdermal fentanyl appears to lack evidence in randomized trials.

Hydromorphone was evaluated in only one study of low methodologic quality (146). Once daily hydromorphone was compared with sustained release oxycodone in participants with chronic pain with or without low back pain. It was considered to be safe and well tolerated for chronic pain, and as being as efficacious as twice-daily sustained-release oxycodone. However, less than $50 \%$ of the patients completed the study, thereby failing to meet the sample size criteria.

Consequently, there is no significant evidence for hydromorphone based on the low quality comparative trial (146) with indeterminate evidence.

Buprenorphine, not commonly used in the United States, has been studied for its role in managing osteoarthritis of the hip and knee, applied transdermally. In a parallel group, comparative trial (155), transdermal buprenorphine was shown to be effective and well-tolerated with analgesic effects similar to tramadol. However, the decrease in the pain scores, though similar in both groups, was only approximately 2 points.

Consequently, the evidence for buprenorphine continues to be poor because of a paucity of randomized trials.

Chou et al $(8,166-168)$ recommended that safe and effective chronic opioid therapy for chronic non-cancer pain requires clinical skills and knowledge in both the principles of opioid prescribing, and in the assessment and management of risks associated with opioid abuse, addiction, and diversion. This recommendation was based on their conclusion of the systematic review that evidence was limited in many areas related to us- 
ing opioids for chronic non-cancer pain. These recommendations are considered by some as biased considering the strong negative recommendations they have provided for other guidelines $(8,166-176)$. It appears that guideline preparers have a different mindset with a priori decisions in favor of opioids and rehabilitation techniques compared with interventional techniques in assessment of pain relief, validity criteria, and outcomes assessment (173-183). These recommendations by Chou et al $(8,166-168)$ and other guideline preparers, (177183) are in contrast to evidence-based medicine (EBM) and comparative effectiveness research principles, guidelines, and applications (32-34,45,166-189). Further, there is evolving evidence for the effectiveness of interventional techniques in multiple areas which has been to some extent ignored by the guideline developers; they have a major focus on lack of evidence, overuse and abuse, even though additional evidence has been rapidly evolving (32-34,169-230).

Noble et al (7) concluded that many patients discontinue long-term oral opioid therapy due to adverse events or insufficient pain relief. They also concluded that there was weak evidence suggesting that patients who continue taking opioids long-term experience clinically significant pain relief. Further, they concluded that whether a patient's quality of life or function improves is inconclusive.

Noble et al (7) expressed significant concern that many participants in the included studies, particularly those treated with orally administered opioids, were so dissatisfied with adverse events or insufficient pain relief that they discontinued participating in the studies. Generally, there is no data provided on these participants after they dropped out of the studies, which makes it impossible to say whether they continued opioid therapy under different protocols or not. We found similar results. Noble et al (7) concluded that for participants able to continue opioids in the studies, evidence (albeit weak) suggests that, for all analyzed models of administration, their pain scores were lower on average than before therapy began, and that this relief could be maintained long-term for over 6 months. However, this data continues to be limited in this systematic review. While all the authors concluded that there was significant improvement in their pain scores, as well as functional status improvement, the application of strict criteria of a $40 \%$ decrease in pain scores or $50 \%$ improvement and significant improvement of functional status of $30 \%$ to $40 \%$ were rarely encountered.

Non-inclusion of observational studies with long-term follow-up may have affected our conclusions. However, with the emerging principles of evidence-based medicine, for this particular review, we decided to analyze the evidence based on randomized trials only. Further, it was our intent to evaluate short- and long-term relief with longterm being over 6 months. There were 3 trials which studied over 6 months $(144,154,165)$. The present systematic review has multiple limitations based on the paucity of randomized placebo-controlled evidence for various types of opioids and multiple conditions they are treated with. Consideration of the observational data may be essential in these circumstances $(1-4,7,15-18,231)$.

Future well-designed research is essential to improve the evidence for opioid therapy in chronic noncancer pain. Clearly, it is important not only that we seek the development of a comprehensive evidence base regarding the effectiveness of opioid pharmacotherapy, but also effective guidance for both prescribing clinicians and governmental policy-makers (232). Clinicians and researchers are called upon to meet the challenge of addressing opioid therapy in a purposeful and coherent manner, rather than continuing to follow the uncoordinated process that has created the present situation. Researchers continue to face a transitional research challenge: problems in clinical practice and at the policy level must guide relevant research at multiple scientific levels including basic science (232).

\subsection{Conclusion}

This systematic review of randomized trials for multiple opioids utilized for managing various chronic pain conditions, showed fair evidence for tramadol in managing osteoarthritis. For all other conditions and all other drugs including tramadol, the evidence was poor based on either weak positive evidence or indeterminate or negative evidence.

\section{Acknowledgments}

The authors wish to thank Sekar Edem for assistance in the search of the literature, Tom Prigge for manuscript review, and Tonie M. Hatton and Diane E. Neihoff, transcriptionists, for their assistance in preparation of this manuscript. We would like to thank the editorial board of Pain Physician for review and criticism in improving the manuscript. 


\section{Author Affiliation}

Dr. Manchikanti is Medical Director of the Pain Management Center of Paducah, Paducah, KY and Associate Clinical Professor, Anesthesiology and Perioperative Medicine, University of Louisville, Louisville, KY .

Dr. Ailinani is Resident of Physical Medicine and Rehabilitation, PGY-4, North Shore-Long Island Jewish Health System.

Dr. Koyyalagunta is Associate Professor and Medical Director of the Pain Management Center at UT MD Anderson Cancer Center -Dept. of Anesthesiology \& Pain Medicine, Houston TX.

Dr. Datta is Director, Vanderbilt University Interventional Pain Program, Associate Professor, Dept. of Anesthesiology, Vanderbilt University Medical Center, Nashville, TN.

Dr. Singh is Medical Director, Pain Diagnostics Associates, Niagara, WI.

Dr. Eriator is Associate Professor of Public Health, Jackson State University, Jackson, MS, Director of the Pain Program, University of Mississippi Medical Center, Jackson, MS.

Dr. Sehgal is the Director of the Interventional Pain Program at the University of Wisconsin School of Medicine and Public Health and Associate Professor, Rehabilitation Medicine, Madison, WI.

Dr. Shah is with the Department of Anesthesiology, Weil-Cornell Medical Center, New York, NY.

Dr. Benyamin is the Medical Director, Millennium Pain Center, Bloomington, IL, Clinical Assistant Professor of Surgery, College of Medicine, University of Illinois, Urbana-Champaign, IL.

Dr. Vallejo is Director of Research, Staff Pain Medicine, Millennium Pain Center, Bloomington, IL, and Adjunct Professor of Biology, Illinois State University, Normal, IL.

Mr. Fellows is Director Emeritus of Psychological Services at the Pain Management Center of Paducah, Paducah, KY.

Dr. Christo is Assistant Professor and Director, Multidisciplinary Pain Fellowship Program Department of Anesthesiology and Critical Care Medicine Division of Pain Medicine, Johns Hopkins University School of Medicine, Baltimore, MD.

\section{References}

1. Manchikanti L, Benyamin R, Datta S, Vallejo R, Smith HS. Opioids in chronic noncancer pain. Expert Rev Neurother 2010; 10:775-789.

2. Trescot AM, Datta S, Glaser S, Sehgal N, Hansen $\mathrm{H}$, Benyamin R, Patel S. Effectiveness of opioids in the treatment of chronic non-cancer pain. Pain Physician 2008; 11:S181-S200.

3. Trescot AM, Helm S, Hansen H, Benyamin R, Adlaka R, Patel S, Manchikanti L. Opioids in the management of chronic non-cancer pain: An update of American Society of Interventional Pain Physicians' (ASIPP) guidelines. Pain Physician 2008; 11:S5-S62.

4. Ballantyne JC. Opioid analgesia: Perspectives on right use and utility. Pain Physician 2007; 10:479-491.

5. Manchikanti L, Singh A. Therapeutic opioids: A ten-year perspective on the complexities and complications of the escalating use, abuse, and nonmedical use of opioids. Pain Physician 2008; 11:S63S88.

6. Manchikanti L, Fellows B, Ailinani $\mathrm{H}$, Pampati V. Therapeutic use, abuse, and nonmedical use of opioids: A tenyear perspective. Pain Physician 2010; 13:401-435.

7. Noble M, Treadwell JR, Tregear SJ, Coates $\mathrm{VH}$, Wiffen PJ, Akafomo C, Schoelles $\mathrm{KM}$. Long-term opioid management for chronic noncancer pain. Cochrane Database Syst Rev 2010; 1:CD006605.

8. Chou R, Huffman L. Use of Chronic Opioid Therapy in Chronic Noncancer Pain: Evidence Review. American Pain Society; Glenview, IL: 2009.

www.ampainsoc.org/pub/pdf/Opioid Final_Evidence_Report.pdf

9. Manchikanti L. Singh V, Datta S, Cohen SP, Hirsch JA. Comprehensive review of epidemiology, scope, and impact of spinal pain. Pain Physician 2009; 12:E35E70.

10. Manchikanti L, Boswell MV, Singh $V$, Benyamin RM, Fellows B, Abdi S, Buenaventura RM, Conn A, Datta S, Derby R, Falco FJE, Erhart S, Diwan S, Hayek SM, Helm S, Parr AT, Schultz DM, Smith HS, Wolfer LR, Hirsch JA. Comprehensive evidence-based guidelines for interventional techniques in the management of chronic spinal pain. Pain Physician 2009; 12:699-802.

11. Bressler HB, Keyes WJ, Rochon PA, Badley $E$. The prevalence of low back pain in the elderly. A systematic review of the literature. Spine (Phila Pa 1976) 1999; 24:1813-1819.

12. Leigh JP, Sheetz RM. Prevalence of back pain among full-time United States workers. BrJ In Med 1989; 46:651-657.

13. Pahor M, Guralnik JM, Wan JY. Lower body osteoarticular pain and dose of analgesic medications in older disabled women: The Women's Health and Aging Study. Am J Public Health 1999; 89:930934.

14. Mailis-Gagnon A, Nicholson K, Yegneswaran B, Zurowski M. Pain characteristics of adults 65 years of age and older referred to a tertiary care pain clinic. Pain Res Manage 2008; 13:389-394.

15. The American Academy of Pain Medicine, the American Pain Society. The use of opioids for the treatment of chronic pain. A consensus statement from the American Academy of Pain Medicine and the American Pain Society. Clin J Pain 
1997; 13:6-8.

16. British Pain Society. Opioids for persistent pain: Good practice. A consensus statement prepared on behalf of the British Pain Society, the Faculty of Pain Medicine and the Royal College of Anaesthetists, the Royal College of General Practitioners and the Faculty of Addictions of the Royal College of Psychiatrists. The British Pain Society, London, UK, January 2010.

17. Jovey RD, Ennis J, Gardner-Nix J, Goldman B, Hays H, Lynch M, Moulin D; Canadian Pain Society. Use of opioid analgesics for the treatment of chronic noncancer pain--a consensus statement and guidelines from the Canadian Pain Society, 2002. Pain Res Manage 2003; 8:3A-28A.

18. Martell BA, O'Connor PG, Kerns RD, Beck WC, Morales KH, Kosten TR, Fiellen DA. Systematic review: Opioid treatment for chronic back pain: Prevalence, efficacy, and association with addiction. Ann Intern Med 2007; 146:116-127.

19. Chou R, Clark E, Helfand M. Comparative efficacy and safety of long-acting oral opioids for chronic non-cancer pain: A systematic review. J Pain Symptom Manage 2003; 26:1026-1048.

20. Kalso E, Edwards JE, Moore RA, McQuay $H J$. Opioids in chronic non-cancer pain: Systematic review of efficacy and safety. Pain 2004; 112:372-380.

21. Furlan AD, Sandoval JA, Mailis-Gagnon A, Tunks E. Opioids for chronic noncancer pain: A meta-analysis of effectiveness and side effects. Can Med Assoc 2006; 174:1589-1594.

22. Eisenberg E, McNicol E, Carr DB. Opioids for neuropathic pain (review). Cochrane Database Syst Rev 2006, 3:CD006146.

23. Deshpande A, Furlan A, Mailis-Gagnon A, Atlas S, Turk D. Opioids for chronic low back pain (review). Cochrane Database Syst Rev 2007; 3:CD004959.

24. Cepeda MS, Camargo F, Zea CLV. Tramadol for osteoarthritis. Cochrane Database Syst Rev 2006 3:CD005522.

25. Devulder J, Richarz U, Nataraja SH. Impact of long-term use of opioids on quality of life in patients with chronic, non-malignant pain. Curr Med Res Opin 2005; 21:1555-1568.

26. Hollingshead J, Duhmke R, Cornblath D. Tramadol for neuropathic pain. Cochrane Database Syst 2006; 3:CD003726.

27. Moore RA, McQuay H. Prevalence of opioid adverse events in chronic non-malignant pain: Systematic review of ran- domised trials of oral opioids. Arthritis Res Ther 2005; 7:R1046-R1051.

28. Dole V.What we have learned from three decades of methadone maintenance treatment. Drug \& Alcohol Review 1994; 13:3-4.

29. Brecher E, Editors of Consumer Reports Magazine. Chapter 15. How well does methadone maintenance work? In: The Consumers Union Report on Licit and IIlicit Drugs. Schaffer Library of Drug Policy, 1972. www.druglibrary.org/schaffer/ library/studies/cu/CU15.html

30. Tennant FS J, Uelman GF. Narcotic maintenance for chronic pain: Medical and legal guidelines. Postgrad Med 1983; 73:81-94.

31. Bouckoms AJ, Masand P, Murray GB, Cassem EH, Stern TA, Tesar GE. Chronic nonmalignant pain treatment with long-term analgesics. Ann Clin Psychiatry 1992; 4:185-192.

32. Manchikanti L. Evidence-based medicine, systematic reviews, and guidelines in interventional pain management: Part 1: Introduction and general considerations. Pain Physician 2008; 11:161-186.

33. Manchikanti L, Hirsch JA, Smith HS. Evidence-based medicine, systematic reviews, and guidelines in interventional pain management: Part 2: Randomized controlled trials. Pain Physician 2008; 11:717-773.

34. Manchikanti L, Benyamin RM, Helm S, Hirsch JA. Evidence-based medicine, systematic reviews, and guidelines in interventional pain management: Part 3: Systematic reviews and meta-analysis of randomized trials. Pain Physician 2009; 12:35-72.

35. Moher D, Cook DJ, Eastwood S, Olkin I, Rennie D, Stroup DF. Improving the quality of reports of meta-analyses of randomised controlled trials: The QUOROM statement. Quality of reporting of meta-analyses. Lancet 1999; 354:18961900.

36. Liberati A, Altman DG, Tetzlaff J, Mulrow C, Gøtzsche PC, loannidis JP, Clarke M, Devereaux PJ, Kleijnen J, Moher D. The PRISMA statement for reporting systematic reviews and meta-analyses of studies that evaluate health care interventions: Explanation and elaboration. Ann Intern Med 2009; 151:W65-W94.

37. van Tulder $M$, Furlan $A$, Bombardier C, Bouter L; Editorial Board of the Cochrane Collaboration Back Review Group. Updated method guidelines for systematic reviews in the Cochrane Collaboration Back Review Group. Spine (Phila Pa 1976) 2003; 28:1290-1299.

38. Jadad AR, Moore RA, Carroll $D$, Jenkinson C, Reynolds DJ, Gavaghan DJ, McQuay HJ. Assessing the quality of reports of randomized clinical trials: Is blinding necessary? Control Clin Trials 1996; 17:1-12.

39. DerSimonian R, Laird N. Meta-analysis in clinical trials. Control Clin Trials 1986; 7:177-188.

40. Altman DG, Schulz KF, Moher D, Egger M, Davidoff F, Elbourne D, Gøtzsche PC, Lang T; CONSORT GROUP (Consolidated Standards of Reporting Trials). The revised CONSORT statement for reporting randomized trials: Explanation and elaboration. Ann Intern Med 2001; 134:663-694.

41. Moher D, Hopewell S, Schulz KF, Montori V, Gøtzsche PC, Devereaux PJ, Elbourne D, Egger M, Altman DG. CONSORT 2010 explanation and elaboration: Updated guidelines for reporting parallel group randomised trials. BMJ 2010; 340:c869.

42. Higgins JP, Thompson SG, Deeks JJ, Altman DG. Measuring inconsistency in meta-analyses. BMJ 2003; 327:557560.

43. Farrar JT. What is clinically meaningful: Outcome measures in pain clinical trials. Clin J Pain 2000; 16:S106-S112.

44. Salaffi F, Stancati A, Silvestri CA, Ciapetti A, Grassi W. Minimal clinically important changes in chronic musculoskeletal pain intensity measured on a numerical rating scale. Eur J Pain 2004; 8:283-291.

45. Manchikanti L, Singh V, Smith HS, Hirsch JA. Evidence-based medicine, systematic reviews, and guidelines in interventional pain management: Part 4: Observational studies. Pain Physician 2009; 12:73-108.

46. Hagg O, Fritzell P, Nordwall A. The clinical importance of changes in outcome scores after treatment for chronic low back pain. Eur Spine J 2003; 12:12-20.

47. Carragee EJ, Chen I. Minimum acceptable outcomes after lumbar spinal fusion. Spine J 2010; 10:313-320.

48. Gatchel RJ, Mayer TG. Testing minimal clinically important difference: Consensus or conundrum? Spine J 2010; 10:321327.

49. Manchikanti L, Singh V, Falco FJE, Cash KA, Pampati V. Evaluation of lumbar facet joint nerve blocks in managing chronic low back pain: A randomized, doubleblind, controlled trial with a 2-year fol- 
low-up. Int J Med Sci 2010; 7:124-135.

50. Manchikanti L, Singh V, Falco FJE, Cash $\mathrm{KA}$, Fellows B. Comparative outcomes of a 2-year follow-up of cervical medial branch blocks in management of chronic neck pain: A randomized, double-blind controlled trial. Pain Physician 2010; 437-450.

51. Harbord R, Higgins J. METAREG: Stata module to perform meta-analysis regression. Boston College Dept of Econ, Boston, MA. www.econpapers.org/software/bocbocode/s446201.htm.

52. SPSS Statistics Software Standard Version (for Windows). Version 9.0.1, Chicago, IL: SPSS Inc; 1999.

53. Huedo-Medina TB, Sánchez-Meca J, Marín-Martínez F, Botella J. Assessing heterogeneity in meta-analysis: $\mathrm{Q}$ statistic or 12 index? Psychol Methods 2006; 11:193-206.

54. Harris RP, Helfand M, Woolf $\mathrm{SH}$, Lohr KN, Mulrow CD, Teutsch SM, Atkins D; Methods Work Group, Third US Preventive Services Task Force. Current methods of the US Preventive Services Task Force. Am J Prevent Med 2001; 20:21-35.

55. Adler L, McDonald C, O'Brien C, Wilson $\mathrm{M}$. A comparison of once-daily tramadol with normal release tramadol in the treatment of pain in osteoarthritis. J Rheumatol 2002; 29:2196-2199.

56. Aqua K, Gimbel JS, Singla N, Ma T, Ahdieh $\mathrm{H}$, Kerwin R. Efficacy and tolerability of oxymorphone immediate release for acute postoperative pain after abdominal surgery: A randomized, doubleblind, active- and placebo-controlled, parallel-group trial. Clin Ther 2007; 29:1000-1012.

57. Aurilio $C$, Pace MC, Passavanti MB, Paladini $A$, Maisto $M$, lannotti $M$, Pota $V$, D'amora $E$, Sansone $P$, Barbarisi $M$. Treatment of ischemic pain in patients suffering from peripheral vasculopathy with transdermal buprenorphine plus epidural morphine with ropivacaine vs. epidural morphine with ropivacaine. Pain Pract 2009; 9:105-114.

58. Beaulieu AD, Peloso P, Bensen W, Clark AJ, Watson CP, Gardner-Nix J, Thomson G, Piraino PS, Eisenhoffer J, Harsanyi Z, Darke AC. A randomized, double-blind, 8-week crossover study of once-daily controlled-release tramadol versus immediate-release tramadol taken as needed for chronic noncancer pain. Clin Ther 2007; 29:49-60.

59. Beaulieu AD, Peloso PM, Haraoui B, Bensen W, Thomson G, Wade J, Quigley
P, Eisenhoffer J, Harsanyi Z, Darke AC. Once-daily, controlled-release tramadol and sustained-release diclofenac relieve chronic pain due to osteoarthritis: A randomized controlled trial. Pain Res Manag 2008; 13:103-110.

60. Bodalia B, McDonald CJ, Smith KJ, O'Brien C, Cousens L. A comparison of the pharmacokinetics, clinical effica$c y$, and tolerability of once-daily tramadol tablets with normal release tramadol capsules. I Pain Symptom Manage 2003: 25:142-149.

61. Caldwell JR, Hale ME, Boyd RE, Hague JM, Iwan T, Shi M, Lacouture PG. Treatment of osteoarthritis pain with controlled release oxycodone or fixed combination oxycodone plus acetaminophen added to nonsteroidal antiinflammatory drugs: A double blind, randomized, multicenter, placebo controlled trial. J Rheumatol 1999; 26:862-869.

62. Caldwell JR, Rapoport RJ, Davis JC, Offenberg HL, Marker HW, Roth SH, Yuan W, Eliot L, Babul N, Lynch PM. Efficacy and safety of a once-daily morphine formulation in chronic, moderate-to-severe osteoarthritis pain: Results from a randomized, placebo-controlled, doubleblind trial and an open-label extension trial. J Pain Symptom Manage 2002; 23:278-291.

63. Chang AK, Bijur PE, Baccelieri A, Gallagher EJ. Efficacy and safety profile of a single dose of hydromorphone compared with morphine in older adults with acute, severe pain: A prospective, randomized, double-blind clinical trial. Am J Geriatr Pharmacother 2009; 7:110.

64. Chindalore VL, Craven RA, Yu KP, Butera PG, Burns LH, Friedmann N. Adding ultralow-dose naltrexone to oxycodone enhances and prolongs analgesia: $A$ randomized, controlled trial of Oxytrex. J Pain 2005; 6:392-399.

65. Cowan DT, Wilson-Barnett J, Griffiths $P$ Vaughan DJ, Gondhia A, Allan LG. A randomized, double-blind, placebo controlled, cross-over pilot study to assess the effects of long-term opioid drug consumption and subsequent abstinence in chronic noncancer pain patients receiving controlled-release morphine. Pain Med 2005; 6:113-121.

66. Daniels SE, Upmalis D, Okamoto A, Lange C, Häeussler J. A randomized, double-blind, phase III study comparing multiple doses of tapentadol IR, oxycodone IR, and placebo for postoperative (bunionectomy) pain. Curr Med Res
Opin 2009; 25:765-776.

67. Daniels S, Casson E, Stegmann JU, Oh C, Okamoto A, Rauschkolb C, Upmalis D. A randomized, double-blind, placebo-controlled phase 3 study of the relative efficacy and tolerability of tapentadol IR and oxycodone IR for acute pain. Curr Med Res Opin 2009; 25:1551-1561.

68. Etropolski MS, Okamoto A, Shapiro DY, Rauschkolb C. Dose conversion between tapentadol immediate and extended release for low back pain. Pain Physician 2010; 13:61-70.

69. Frank B, Serpell MG, Hughes J, Matthews JN, Kapur D. Comparison of analgesic effects and patient tolerability of nabilone and dihydrocodeine for chronic neuropathic pain: Randomised, crossover, double blind study. BMJ 2008; 336:199-201

70. Gatti A, Reale C, Occhioni R, Luzi M, Canneti A, De Polo C, Gubernari M, Mammucari M, Fabrizio Sabato A. Standard therapy with opioids in chronic pain management: ORTIBER study. Clin Drug Investig 2009; 29:17-23.

71. Gilron I, Bailey JM, Tu D, Holden RR, Weaver DF, Houlden RL. Morphine, gabapentin, or their combination for neuropathic pain. N Engl / Med 2005; 352:1324-1334.

72. Gimbel JS, Richards P, Portenoy RK. Controlled-release oxycodone for pain in diabetic neuropathy: A randomized controlled trial. Neurology 2003; 60:927934.

73. Gordon A, Rashiq S, Moulin DE, Clark $A J$, Beaulieu AD, Eisenhoffer J, Piraino PS, Quigley P, Harsanyi Z, Darke AC. Buprenorphine transdermal system for opioid therapy in patients with chronic low back pain. Pain Res Manag 2010; 15:169-178.

74. Gordon A, Callaghan D, Spink D, Cloutier C, Dzongowski P, O'Mahony W, Sinclair D, Rashiq S, Buckley N, Cohen G, Kim J, Boulanger A, Piraino PS, Eisenhoffer J, Harsanyi Z, Darke AC, Michalko $\mathrm{KJ}$. Buprenorphine transdermal system in adults with chronic low back pain: A randomized, double-blind, placebocontrolled crossover study, followed by an open-label extension phase. Clin Ther 2010; 32:844-860.

75. Gould EM, Jensen MP, Victor TW, Gammaitoni AR, White RE, Galer BS. The pain quality response profile of oxymorphone extended release in the treatment of low back pain. Clin J Pain 2009; 25:116-122. 
76. Grosset $A B$, Roberts $M S$, Woodson $M E$, Shi M, Swanton RE, Reder RF, Buckley BJ. Comparative efficacy of oral extended-release hydromorphone and immediate-release hydromorphone in patients with persistent moderate to severe pain: Two randomized controlled trials. J Pain Symptom Manage 2005; 29:584-594.

77. Hale ME, Speight K, Harsanyi Z, Iwan T, Slagle N, Lacouture P, Darke A. Efficacy of 12 hourly controlled-release codeine compared with as required dosing of acetaminophen plus codeine in patients with chronic low back pain. Pain Res Manag 1997; 2:33-38.

78. Hale ME, Dvergsten C, Gimbel J. Efficacy and safety of oxymorphone extended release in chronic low back pain: Results of a randomized, double-blind, placebo- and active-controlled phase III study. J Pain 2005; 6:21-28.

79. Hale ME, Fleischmann R, Salzman R, Wild J, Iwan T, Swanton RE, Kaiko RF, Lacouture PG. Efficacy and safety of controlled-release versus immediate-release oxycodone: Randomized, doubleblind evaluation in patients with chronic back pain. Clin J Pain 1999; 15:179-183.

80. Hale ME, Tudor IC, Khanna S, Thipphawong J. Efficacy and tolerability of oncedaily OROS hydromorphone and twicedaily extended-release oxycodone in patients with chronic, moderate to severe osteoarthritis pain: Results of a 6week, randomized, open-label, noninferiority analysis. Clin Ther 2007; 29:874888.

81. Hamann S, Sloan P. Oral naltrexone to enhance analgesia in patients receiving continuous intrathecal morphine for chronic pain: A randomized, doubleblind, prospective pilot study. I Opioid Manag 2007; 3:137-144.

82. Harati $Y$, Gooch $C$, Swenson M, Edelman SV, Greene D, Raskin P, Donofrio P, Cornblath D, Olson WH, Kamin M. Maintenance of the long-term effectiveness of tramadol in treatment of the pain of diabetic neuropathy. J Diabetes Complications 2000; 14:65-70.

83. Harke H, Gretenkort P, Ladleif HU, Rahman $\mathrm{S}$, Harke $\mathrm{O}$. The response of neuropathic pain and pain in complex regional pain syndrome I to carbamazepine and sustained-release morphine in patients pretreated with spinal cord stimulation: A double-blinded randomized study. Anesth Analg 2001; 92:488-495.

84. Hartrick C, Van Hove I, Stegmann JU, Oh
C, Upmalis D. Efficacy and tolerability of tapentadol immediate release and oxycodone $\mathrm{HCl}$ immediate release in patients awaiting primary joint replacement surgery for end-stage joint disease: A 10-day, phase III, randomized, double-blind, active- and placebo-controlled study. Clin Ther 2009; 31:260271.

85. Huse E, Larbig W, Flor $\mathrm{H}$, Birbaumer $\mathrm{N}$. The effect of opioids on phantom limb pain and cortical reorganization. Pain 2001; 90:47-55.

86. James IG, O'Brien CM, McDonald CJ. A randomized, double-blind, doubledummy comparison of the efficacy and tolerability of low-dose transdermal buprenorphine (BuTrans seven-day patches) with buprenorphine sublingual tablets (Temgesic) in patients with osteoarthritis pain. J Pain Symptom Manage 2010; 40:266-278.

87. Jensen EM, Ginsberg F. Tramadol versus dextropropoxyphene in the treatment of osteoarthritis: A short term doubleblind study. Drug Invest 1994; 8:211218.

88. Kalso E, Simpson KH, Slappendel R, Dejonckheere J, Richarz U. Predicting longterm response to strong opioids in patients with low back pain: Findings from a randomized, controlled trial of transdermal fentanyl and morphine. BMC Med 2007; 5:39.

89. Katz N, Sun S, Johnson F, Stauffer J. ALO01 (morphine sulfate and naltrexone hydrochloride) extended-release capsules in the treatment of chronic pain of osteoarthritis of the hip or knee: Pharmacokinetics, efficacy, and safety. J Pain 2010; 11:303-311.

90. Khoromi S, Cui L, Nackers L, Max MB. Morphine, nortriptyline and their combination vs. placebo in patients with chronic lumbar root pain. Pain 2007; 130:66-75.

91. Kivitz A, Ma C, Ahdieh H, Galer BS. A 2week, multicenter, randomized, doubleblind, placebo-controlled, dose-ranging, phase III trial comparing the efficacy of oxymorphone extended release and placebo in adults with pain associated with osteoarthritis of the hip or knee. Clin Ther 2006; 28:352-364.

92. Kleinert $R$, Lange $C$, Steup A, Black $P$, Goldberg J, Desjardins P. Single dose analgesic efficacy of tapentadol in postsurgical dental pain: The results of a randomized, double-blind, placebocontrolled study. Anesth Analg 2008;
107:2048-2055.

93. Landau CJ, Carr WD, Razzetti AJ, Sessler NE, Munera C, Ripa SR. Buprenorphine transdermal delivery system in adults with persistent noncancer-related pain syndromes who require opioid therapy: A multicenter, 5-week runin and randomized, double-blind maintenance-of-analgesia study. Clin Ther 2007; 29:2179-2193.

94. Lange B, Kuperwasser B, Okamoto A, Steup A, Häufel T, Ashworth J, Etropolski M. Efficacy and safety of tapentadol prolonged release for chronic osteoarthritis pain and low back pain. Adv Ther 2010; 27:381-399.

95. Langford R, McKenna F, Ratcliffe S, Vojtassák J, Richarz U. Transdermal fentanyl for improvement of pain and functioning in osteoarthritis: A randomized, placebo-controlled trial. Arth Rheum 2006; 54:1829-1837.

96. Likar R, Lorenz V, Korak-Leiter M, Kager I, Sittl R. Transdermal buprenorphine patches applied in a 4-day regimen versus a 3-day regimen: A singlesite, Phase III, randomized, open-label, crossover comparison. Clin Ther 2007; 29:1591-1606.

97. Litkowski LJ, Christensen SE, Adamson DN, Van Dyke T, Han SH, Newman KB. Analgesic efficacy and tolerability of oxycodone $5 \mathrm{mg} /$ ibuprofen 400 mg compared with those of oxycodone $5 \mathrm{mg} /$ acetaminophen $325 \mathrm{mg}$ and hydrocodone $7.5 \mathrm{mg} /$ acetaminophen $500 \mathrm{mg}$ in patients with moderate to severe postoperative pain: A randomized, double-blind, placebo-controlled, single-dose, parallel-group study in a dental pain model. Clin Ther 2005; 27:418429.

98. Ma K, Jiang W, Zhou Q, Du DP. The efficacy of oxycodone for management of acute pain episodes in chronic neck pain patients. Int J Clin Pract 2008; 62:241247.

99. Malonne H, Coffiner M, Sonet B, Sereno A, Vanderbist F. Efficacy and tolerability of sustained-release tramadol in the treatment of symptomatic osteoarthritis of the hip or knee: A multicenter, randomized, double-blind, placebo-controlled study. Clin Ther 2004; 26:17741782.

100. Malonne $H$, Coffiner $M$, Fontaine $D$, Sonet $B$, Sereno A, Peretz A, Vanderbist F. Long-term tolerability of tramadol LP, a new once-daily formulation, in patients with osteoarthritis or low back pain. J 
Clin Pharm Ther 2005; 30:113-120.

101. Matsumoto AK, Babul N, Ahdieh H. Oxymorphone extended-release tablets relieve moderate to severe pain and improve physical function in osteoarthritis: results of a randomized, doubleblind, placebo- and active-controlled phase III trial. Pain Med 2005; 6:357366.

102. Max MB, Schafer SC, Culnane M, Dubner $\mathrm{R}$, Gracely RH. Association of pain relief with drug side effects in postherpetic neuralgia: A single-dose study of clonidine, codeine, ibuprofen, and placebo. Clin Pharmacol Ther 1988; 43:363-371.

103. Mcllwain $\mathrm{H}$, Ahdieh H. Safety, tolerability, and effectiveness of oxymorphone extended release for moderate to severe osteoarthritis pain: A one-year study. Am J Ther 2005; 12:106-112.

104. Morley JS, Bridson J, Nash TP, Miles JB, White S, Makin MK. Low-dose methadone has an analgesic effect in neuropathic pain: A double-blind randomized controlled crossover trial. Palliat Med 2003; 17:576-587.

105. Moulin DE, lezzi $A$, Amireh $R$, Sharpe WK, Boyd D, Merskey H. Randomised trial of oral morphine for chronic noncancer pain. Lancet 1996; 347:143-147.

106. Mullican WS, Lacy JR; TRAMAP-ANAG006 Study Group. Tramadol/acetaminophen combination tablets and codeine/ acetaminophen combination capsules for the management of chronic pain: A comparative trial. Clin Ther 2001; 23:1429-1445.

107. Munera C, Drehobl M, Sessler NE, Landau C. A randomized, placebo-controlled, double-blinded, parallel-group, 5-week study of buprenorphine transdermal system in adults with osteoarthritis. J Opioid Manag 2010; 6:193-202.

108. Nicholson B, Ross E, Weil A, Sasaki J, Sacks G. Treatment of chronic moderate-to-severe non-malignant pain with polymer-coated extended-release morphine sulfate capsules. Curr Med Res Opin 2006; 22:539-550.

109. Niemann T, Madsen LG, Larsen S, Thorsgaard N. Opioid treatment of painful chronic pancreatitis. Int I Pancreatol 2000; 27:235-240.

110. Norrbrink C, Lundeberg T. Tramadol in neuropathic pain after spinal cord injury: A randomized, double-blind, placebo-controlled trial. Clin J Pain 2009; 25:177-184.

111. Palangio M, Morris E, Doyle RT Jr, Dornseif $\mathrm{BE}$, Valente TJ. Combination hydro- codone and ibuprofen versus combination oxycodone and acetaminophen in the treatment of moderate or severe acute low back pain. Clin Ther 2002 24:87-99.

112. Parris WC, Johnson BW Jr, Croghan MK, Moore MR, Khojasteh A, Reder RF, Kaiko RF, Buckley BJ. The use of controlledrelease oxycodone for the treatment of chronic cancer pain: A randomized, double-blind study. J Pain Symptom Manage 1998; 16:205-211

113. Paulson DM, Kennedy DT, Donovick RA, Carpenter RL, Cherubini M, Techner L Du W, Ma Y, Schmidt WK, Wallin B, Jackson D. Alvimopan: An oral, peripherally acting, mu-opioid receptor antagonist for the treatment of opioid-induced bowel dysfunction--a 21-day treatmentrandomized clinical trial. J Pain 2005 6:184-192.

114. Perrot $S$, Krause $D$, Crozes $P$, Naïm $C_{\text {; }}$ GRTF-ZAL-1 Study Group. Efficacy and tolerability of paracetamol/tramadol (325 mg/37.5 mg) combination treatment compared with tramadol $(50 \mathrm{mg})$ monotherapy in patients with subacute low back pain: A multicenter, randomized, double-blind, parallel-group, 10 day treatment study. Clin Ther 2006; 28:1592-1606.

115. Petrone D, Kamin M, Olson W. Slowing the titration rate of tramadol $\mathrm{HCl}$ reduces the incidence of discontinuation due to nausea and/or vomiting: A doubleblind randomized trial. J Clin Pharm Ther 1999; 24:115-123.

116. Portenoy RK, Messina J, Xie F, Peppin J. Fentanyl buccal tablet (FBT) for relief of breakthrough pain in opioid-treated patients with chronic low back pain: A randomized, placebo-controlled study. Curr Med Res Opin 2007; 23:223-233.

117. Raber M, Hofmann S, Junge K, Momberger $H$, Kuhn D. Analgesic efficacy and tolerability of tramadol $100 \mathrm{mg}$ sustained-release capsules in patients with moderate to severe chronic low back pain. Clin Drug Inves 1999; 17:415-423.

118. Raja SN, Haythornthwaite JA, Pappagallo M, Clark MR, Travison TG, Sabeen S, Royall RM, Max MB. Opioids versus antidepressants in postherpetic neuralgia: A randomized, placebo-controlled trial. Neurology 2002; 59:1015-1021.

119. Ralphs JA, Williams AC, Richardson PH, Pither CE, Nicholas MK. Opiate reduction in chronic pain patients: A comparison of patient-controlled reduction and staff controlled cocktail methods. Pain 1994; 56:279-288.
120. Rauck RL, Bookbinder SA, Bunker TR, Alftine CD, Ghalie R, Negro-Vilar A, de Jong E, Gershon S. The ACTION study: a randomized, open-label, multicenter trial comparing once-a-day extended-release morphine sulfate capsules (AVINZA) to twice-a-day controlled-release oxycodone hydrochloride tablets (OxyContin) for the treatment of chronic, moderate to severe low back pain. J Opioid Manag 2006; 2:155-166. Erratum in: J Opioid Manag 2006; 2:276.

121. Roth SH, Fleischmann RM, Burch FX, Dietz F, Bockow B, Rapoport RJ, Rutstein J, Lacouture PG. Around-the-clock, controlled-release oxycodone therapy for osteoarthritis-related pain: Placebocontrolled trial and long-term evaluation. Arch Intern Med 2000; 160:853860.

122. Rowbotham MC, Twilling L, Davies PS, Reisner L, Taylor K, Mohr D. Oral opioid therapy for chronic peripheral and central neuropathic pain. $N$ Engl J Med 2003; 348:1223-1232.

123. Ruoff GE. Slowing the initial titration rate of tramadol improves tolerability. Pharmacother 1999; 19:88-93.

124. Ruoff $G E$, Rosenthal N, Jordan D, Karim R, Kamin M; Protocol CAPSS-112 Study Group. Tramadol/acetaminophen combination tablets for the treatment of chronic lower back pain: A multicenter, randomized, double-blind, placebo-controlled outpatient study. Clin Ther 2003; 25:1123-1141.

125. Salzman RT, Roberts MS, Wild J, Fabian C, Reder RF, Goldenheim PD. Can a controlled release oral dose form of oxycodone be used as readily as an immediate release form for the purpose of titrating to stable pain control? J Pain Sympt Manage 1999; 18:271-279.

126. Sandner-Kiesling $A$, Leyendecker $P$, Hopp M, Tarau L, Lejcko J, Meissner W, Sevcik P, Hakl M, Hrib R, Uhl R, Dürr H, Reimer K. Long-term efficacy and safety of combined prolonged-release oxycodone and naloxone in the management of non-cancer chronic pain. Int J Clin Pract 2010; 64:763-774.

127. Simpson DM, Messina J, Xie F, Hale ME. Fentanyl buccal tablet for the relief of breakthrough pain in opioid-tolerant adult patients with chronic neuropathic pain: A multicenter, randomized, double-blind, placebo-controlled study. Clin Ther 2007; 29:588-601.

128. Sindrup $\mathrm{SH}$, Madsen $\mathrm{C}$, Brøsen $\mathrm{K}$, Jensen TS. The effect of tramadol in pain- 
ful polyneuropathy in relation to serum drug and metabolite levels. Clin Pharmacol Ther 1999; 66:636-641.

129. Sindrup SH, Andersen G, Madsen C, Smith T, Brøsen K, Jensen TS. Tramadol relieves pain and allodynia in polyneuropathy: A randomised, double-blind, controlled trial. Pain 1999; 83:85-90.

130. Sorge J, Stadler T. Comparison of the analgesic efficacy and tolerability of tramadol $100 \mathrm{mg}$ sustained-release tablets and tramadol $50 \mathrm{mg}$ capsules for the treatment of chronic low back pain. Clin Drug Invest 1997; 14:157-164.

131. Sorge J, Sittl R. Transdermal buprenorphine in the treatment of chronic pain: Results of a phase III, multicenter, randomized, double-blind, placebo-controlled study. Clin Ther 2004; 26:18081820.

132. Stegmann JU, Weber H, Steup A, Okamoto A, Upmalis D, Daniels S. The efficacy and tolerability of multiple-dose tapentadol immediate release for the relief of acute pain following orthopedic (bunionectomy) surgery. Curr Med Res Opin 2008; 24:3185-3196.

133. Tessaro L, Bandieri E, Costa G, Fornasier G, lorno V, Pizza C, Pastacaldi G, Micheletto $G$. Use of oxycodone controlledrelease immediately after NSAIDs: A new approach to obtain good pain control. Eur Rev Med Pharmacol Sci 2010; 14:113-121.

134. Thorne C, Beaulieu AD, Callaghan DJ, O'Mahony WF, Bartlett JM, Knight R, Kraag GR, Akhras R, Piraino PS, Eisenhoffer J, Harsanyi Z, Darke AC. A randomized, double-blind, crossover comparison of the efficacy and safety of oral controlled-release tramadol and placebo in patients with painful osteoarthritis. Pain Res Manag 2008; 13:93-102.

135. Vorsanger $G$, Xiang J, Jordan D, Farrell J. Post hoc analysis of a randomized, double-blind, placebo-controlled efficacy and tolerability study of tramadol extended release for the treatment of osteoarthritis pain in geriatric patients. Clin Ther 2007; 29:S2520-S2535.

136. Vorsanger $G$, Xiang J, Okamoto A, Upmalis D, Moskovitz B. Evaluation of study discontinuations with tapentadol immediate release and oxycodone immediate release in patients with low back or osteoarthritis pain. J Opioid Manage 2010; 6:169-179.

137. Wallace M, Skowronski R, Khanna S, Tudor IC, Thipphawong J. Efficacy and safety evaluation of once-daily OROS hydro- morphone in patients with chronic low back pain: A pilot open-label study (DO127). Curr Med Res Opin 2007; 23:981999.

138. Watson CP, Babul N. Efficacy of oxycodone in neuropathic pain: a randomized trial in postherpetic neuralgia. Neurology 1998; 50:1837-1841.

139. Watson CP, Moulin D, Watt-Watson J, Gordon A, Eisenhoffer J. Controlled-release oxycodone relieves neuropathic pain: A randomized controlled trial in painful diabetic neuropathy. Pain 2003; 105:71-78.

140. Webster $L$, Jansen JP, Peppin J, Lasko $B$, Irving G, Morlion B, Snidow J, Pierce A, Mortensen E, Kleoudis C, Carter E. Alvimopan, a peripherally acting mu-opioid receptor (PAM-OR) antagonist for the treatment of opioid-induced bowel dysfunction: Results from a randomized, double-blind, placebo-controlled, dose finding study in subjects taking opioids for chronic non-cancer pain. Pain 2008; 137:428-440.

141. Wilder-Smith $\mathrm{CH}$, Hill L, Spargo K, Kalla A. Treatment of severe pain from osteoarthritis with slow-release tramadol or dihydrocodeine in combination with NSAID's: A randomised study comparing analgesia, antinociception and gastrointestinal effects. Pain 2001; 91:2331.

142. Zautra AJ, Smith BW. Impact of controlled release oxycodone on efficacy beliefs and coping efforts among osteoarthritis patients with moderate to severe pain. Clin J Pain 2005; 21:471-477.

143. Afilalo M, Etropolski MS, Kuperwasser B, Kelly K, Okamoto A, Van Hove I, Steup A, Lange B, Rauschkolb C, Haeussler J. Efficacy and safety of tapentadol extended release compared with oxycodone controlled release for the management of moderate to severe chronic pain related to osteoarthritis of the knee: $\mathrm{A}$ randomized, double-blind, placebo- and active-controlled phase III study. Clin Drug Investig 2010; 30:489-505.

144. Allan L, Richarz U, Simpson K, Slappendel R. Transdermal fentanyl versus sustained release oral morphine in strongopioid naïve patients with chronic low back pain. Spine(Phila Pa 1976) 2005; 30:2484-2490.

145. Babul N, Noveck R, Chipman H, Roth SH, Gana T, Albert K. Efficacy and safety of extended-release, once-daily tramadol in chronic pain: A randomized 12-week clinical trial in osteoarthritis of the knee.
J Pain Symptom Manage 2004; 28:5971.

146. Binsfeld H, Szczepanski L, Waechter S, Richarz U, Sabatowski R. A randomized study to demonstrate noninferiority of once-daily $\operatorname{OROS}\left({ }^{\oplus}\right)$ hydromorphone with twice-daily sustained-release oxycodone for moderate to severe chronic noncancer pain. Pain Pract 2010; 10:404-415.

147. Burch F, Fishman R, Messina N, Corser B, Radulescu F, Sarbu A, Craciun-Nicodin MM, Chiriac R, Beaulieu A, Rodrigues J, Beignot-Devalmont $P$, Duplan A, Robertson S, Fortier L, Bouchard S. A comparison of the analgesic efficacy of tramadol Contramid OAD versus placebo in patients with pain due to osteoarthritis. J Pain Symptom Manage 2007; 34:328-338.

148. Buynak R, Shapiro DY, Okamoto A, Van Hove I, Rauschkolb C, Steup A, Lange B, Lange C, Etropolski M. Efficacy and safety of tapentadol extended release for the management of chronic low back pain: Results of a prospective, randomized, double-blind, placebo- and activecontrolled Phase III study. Expert Opin Pharmacother 2010; 11:1787-1804.

149. Galer BS, Lee D, Ma T, Nagle B, Schlagheck TG. MorphiDex (morphine sulfate/dextromethorphan hydrobromide combination) in the treatment of chronic pain: Three multicenter, randomized, double blind, controlled clinical trials fail to demonstrate enhanced opioid analgesia or reduction in tolerance. Pain 2005; 115:284-295.

150. Gana TJ, Pascual ML, Fleming RR, Schein JR, Janagap CC, Xiang J, Vorsanger GJ; 023 Study Group. Extended-release tramadol in the treatment of osteoarthritis: A multicenter, randomized, doubleblind, placebo-controlled clinical trial. Curr Med Res Opin 2006; 22: 1391-1401.

151. Hale ME, Ahdieh H, Ma T, Rauck R; Oxymorphone ER Study Group 1. Efficacy and safety of OPANA ER (oxymorphone extended release) for relief of moderate to severe chronic low back pain in opioid experienced patients: A 12-week, randomized, double-blind, placebo-controlled study. J Pain 2007; 8:175-184.

152. Hale ME, Upmalis $D$, Okamoto $A$, Lange C, Rauschkolb C. Tolerability of tapentadol immediate release in patients with lower back pain or osteoarthritis of the hip or knee over 90 days: A randomized, double-blind study. Curr Med Res Opin 2009; 25:1095-1104. 
153. Hanna M, O'Brien C, Wilson M. Prolonged release oxycodone enhances the effects of existing gabapentin therapy in painful diabetic neuropathy patients. Eur J Pain 2008; 12 804-813.

154. Jamison RN, Raymond SA, Slawsby EA, Nedeljkovic SS, Katz NP. Opioid therapy for chronic noncancer back pain. A randomized prospective study. Spine (Phila Pa 1976) 1998; 23:2591-2600.

155. Karlsson M, Berggren AC. Efficacy and safety of low-dose transdermal buprenorphine patches $(5,10$, and $20 \mathrm{mi}-$ $\mathrm{crog} / \mathrm{h}$ ) versus prolonged-release tramadol tablets $(75,100,150$, and $200 \mathrm{mg})$ in patients with chronic osteoarthritis pain: A 12-week, randomized, open-label, controlled, parallel-group noninferiority study. Clin Ther 2009; 31:503-513.

156. Katz N, Rauck R, Ahdieh H, Ma T, Gerritsen van der Hoop R, Kerwin R, Podolsky G. A 12-week, randomized, placebo-controlled trial assessing the safety and efficacy of oxymorphone extended release for opioid naive patients with chronic low back pain. Curr Med Res Opin 2007; 23:117-128.

157. Markenson JA, Croft J, Zhang PG, Richards $P$. Treatment of persistent pain associated with osteoarthritis with controlled-release oxycodone tablets in a randomized controlled clinical trial. Clin J Pain 2005; 21:524-535.

158. Mongin G, Yakusevich V, Köpe A, Shostak N, Pikhlak E, Popdán L, Simon J, Navarro C, Fortier L, Robertson S, Bouchard S. Efficacy and safety assessment of a novel once-daily tablet formulation of tramadol: A randomised, controlled study versus twice daily tramadol in patients with osteoarthritis of the knee. Clin Drug Invest 2004; 24:545-558.

159. Nicholson B, Ross E, Sasaki J, Weil A. Randomized trial comparing polymercoated extended-release morphine sulphate to controlled-release oxycodone $\mathrm{HCl}$ in moderate to severe nonmalignant pain. Curr Med Res Opin 2006; 22:15031514.

160. Rauck RL, Bookbinder SA, Bunker TR, Alftine CD, Gershon S, de Jong E, NegroVilar A, Ghalie R. A randomized, open-label, multicenter trial comparing once-aday AVINZA (morphine sulfate extended-release capsules) versus twice-aday OxyContin (oxycodone hydrochloride controlled-released tablets) for the treatment of chronic, moderate to severe low back pain: Improved physical functioning in the ACTION trial. J Opioid Manage 2007; 3:35-43.
161. Simpson K, Leyendecker $P$, Hopp $M$, Müller-Lissner $\mathrm{S}$, Löwenstein $\mathrm{O}$, De Andrés J, Troy Ferrarons J, Bosse B, Krain B, Nichols T, Kremers W, Reimer K. Fixedratio combination oxycodone/naloxone compared with oxycodone alone for the relief of opioid-induced constipation in moderate-to-severe noncancer pain. Curr Med Res Opin 2008; 24:35033512.

162. Vondrackova $D$, Leyendecker $P$, Meissner W, Hopp M, Szombati I, Hermanns K, Ruckes C, Weber S, Grothe B, Fleischer W, Reimer K. Analgesic efficacy and safety of oxycodone in combination with naloxone as prolonged release tablets in patients with moderate to severe chronic pain. J Pain 2008; 9:1144-1154.

163. Vorsanger GJ, Xiang J, Gana TJ, Pascual $M L$, Fleming RR. Extended-release tramadol (tramadol ER) in the treatment of chronic low back pain. J Opioid Manag 2008; 4:87-97.

164. Webster LR, Butera PG, Moran LV, Wu $\mathrm{N}$, Burns LH, Friedmann N. Oxytrex minimizes physical dependence while providing effective analgesia: A randomized controlled trial in low back pain. $J$ Pain 2006; 7:937-946.

165. Wild JE, Grond S, Kuperwasser B, Gilbert J, McCann B, Lange B, Steup A, Häufel T, Etropolski MS, Rauschkolb C, Lange R. Long-term safety and tolerability of tapentadol extended release for the management of chronic low back pain or osteoarthritis pain. Pain Pract 2010; 10:416-427.

166. Chou R, Fanciullo GJ, Fine PG, Adler JA, Ballantyne JC, Davies P, Donovan MI, Fishbain DA, Foley KM, Fudin J, Gilson AM, Kelter A, Mauskop A, O'Connor PG, Passik SD, Pasternak GW, Portenoy RK, Rich BA, Roberts RG, Todd KH, Miaskowski C; American Pain Society-American Academy of Pain Medicine Opioids Guidelines Panel. Clinical guidelines for the use of chronic opioid therapy in chronic noncancer pain. J Pain 2009; 10:113-130.

167. Chou R, Fanciullo GJ, Fine PG, Miaskowski C, Passik SD, Portenoy RK. Opioids for chronic noncancer pain: Prediction and identification of aberrant drug-related behaviors: A review of the evidence for an American Pain Society and American Academy of Pain Medicine clinical practice guideline. J Pain 2009; 10:131-146.

168. Chou R, Ballantyne JC, Fanciullo GJ, Fine PG, Miaskowski C. Research gaps on use of opioids for chronic noncancer pain: Findings from a review of the evidence for an American Pain Society and American Academy of Pain Medicine clinical practice guideline. J Pain 2009; 10:147159.

169. Chou R, Huffman L. Evaluation and Management of Low Back Pain: Evidence Review. American Pain Society, Glenview, IL, 2009.

170. Chou R, Baisden J, Carragee EJ, Resnick DK, Shaffer WO, Loeser JD. Surgery for low back pain: A review of the evidence for an American Pain Society Clinical Practice Guideline. Spine (Phila $\mathrm{Pa}$ 1976) 2009; 34:1094-1109.

171. Chou R, Atlas SJ, Stanos SP, Rosenquist RW. Nonsurgical interventional therapies for low back pain: A review of the evidence for an American Pain Society clinical practice guideline. Spine (Phila Pa 1976) 2009; 34:1078-1093.

172. Chou R, Loeser JD, Owens DK, Rosenquist RW, Atlas SJ, Baisden J, Carragee EJ, Grabois M, Murphy DR, Resnick DK, Stanos SP, Shaffer WO, Wall EM; American Pain Society Low Back Pain Guideline Panel. Interventional therapies, surgery, and interdisciplinary rehabilitation for low back pain: An evidencebased clinical practice guideline from the American Pain Society. Spine (Phila Pa 1976) 2009; 34:1066-1077.

173. Manchikanti L, Datta S, Derby R, Wolfer LR, Benyamin RM, Hirsch JA. A critical review of the American Pain Society clinical practice guidelines for interventional techniques: Part 1. Diagnostic interventions. Pain Physician 2010; 13:E141E174.

174. Manchikanti L, Datta S, Gupta S, Munglani $R$, Bryce DA, Ward SP, Benyamin RM, Sharma ML, Helm II S, Fellows B, Hirsch JA. A critical review of the American Pain Society clinical practice guidelines for interventional techniques: Part 2. Therapeutic interventions. Pain Physician 2010; 13:E215-E264.

175. Chou R. Critiquing the critiques: The American Pain Society guideline and the American Society of Interventional Pain Physicians response to it. Pain Physician 2010; in submission.

176. Manchikanti L. In response: Critiquing the critiques: The American Pain Society guideline and the American Society of Interventional Pain Physicians response to it. Pain Physician 2010; in submission; author reply in submission.

177. American College of Occupational and Environmental Medicine (ACOEM) Low back Disorders. In Occupational Medicine Practice Guidelines: Evaluation and 
Management of Common Health Problems and Functional Recovery of Workers, 2nd Ed. American College of Occupational and Environmental Medicine Press, Elk Grove Village, 2007.

178. American College of Occupational and Environmental Medicine (ACOEM) Chronic Pain. In Occupational Medicine Practice Guidelines: Evaluation and Management of Common Health Problems and Functional Recovery of Workers, Second Edition. American College of Occupational and Environmental Medicine Press, Elk Grove Village, ILm 2008.

179. Manchikanti L, Singh V, Derby R, Helm S, Trescot AM, Staats PS, Prager JP, Hirsch JA. Review of occupational medicine practice guidelines for interventional pain management and potential implications. Pain Physician 2008; 11:271289.

180. Manchikanti L, Singh V, Helm S, Trescot AM, Hirsch JA. A critical appraisal of 2007 American College of Occupational and Environmental Medicine (ACOEM) practice guidelines for interventional pain management: An independent review utilizing AGREE, AMA, IOM, and other criteria. Pain Physician 2008; 11:291-310.

181. Manchikanti L, Singh V, Derby R, Schultz DM, Benyamin RM, Prager JP, Hirsch JA. Reassessment of evidence synthesis of occupational medicine practice guidelines for interventional pain management. Pain Physician 2008; 11:393482.

182. Manchikanti L, Falco FJE, Boswell MV, Hirsch JA. Facts, fallacies, and politics of comparative effectiveness research: Part 1. Basic considerations. Pain Physician 2010; 13:E23-E54.

183. Manchikanti L, Falco FJE, Boswell MV, Hirsch JA. Facts, fallacies, and politics of comparative effectiveness research: Part 2. Implications for interventional pain management. Pain Physician 2010; 13:E55-E79.

184. Manchikanti L, Derby R, Wolfer LR, Singh V, Datta S, Hirsch JA. Evidencebased medicine, systematic reviews, and guidelines in interventional pain management: Part 5. Diagnostic accuracy studies. Pain Physician 2009; 12:517540.

185. Manchikanti L, Datta S, Smith HS, Hirsch JA. Evidence-based medicine, systematic reviews, and guidelines in interventional pain management: Part 6. Systematic reviews and meta-analyses of observational studies. Pain Physician 2009; 12:819-850.

186. Manchikanti L, Derby $R$, Wolfer LR, Singh V, Datta S, Hirsch JA. Evidencebased medicine, systematic reviews, and guidelines in interventional pain management: Part 7: Systematic reviews and meta-analyses of diagnostic accuracy studies. Pain Physician 2009; 12:929-963.

187. Manchikanti L, Singh V, Boswell MV. Interventional pain management at crossroads: The perfect storm brewing for a new decade of challenges. Pain Physician 2010; 13:E111-E140.

188. Benyamin RM, Datta S, Falco FJE. A perfect storm in interventional pain management: Regulated, but unbalanced. Pain Physician 2010; 13:109-116.

189. Manchikanti L, Hirsch JA. Obama health care for all Americans: Practical implications. Pain Physician 2009; 12:289-304.

190. Friedly J, Chan L, Deyo R. Increases in lumbosacral injections in the Medicare population: 1994 to 2001. Spine (Phila Pa 1976) 2007; 32:1754-1760.

191. Manchikanti L, Singh V, Pampati V, Smith HS, Hirsch JA. Analysis of growth of interventional techniques in managing chronic pain in Medicare population: A 10-year evaluation from 1997 to 2006. Pain Physician 2009; 12:9-34.

192. Manchikanti L, Pampati V, Singh V, Boswell MV, Smith HS, Hirsch JA. Explosive growth of facet joint interventions in the Medicare population in the United States: A comparative evaluation of 1997, 2002, and 2006 data. BMC Health Serv Res 2010; 10:84.

193. Manchikanti L, Pampati V, Boswell MV, Smith HS, Hirsch JA. Analysis of the growth of epidural injections and costs in the Medicare population: A comparative evaluation of 1997, 2002, and 2006 data. Pain Physician 2010; 13:199-212.

194. Manchikanti L, Cash KA, McManus CD, Pampati V, Singh V, Benyamin RM. The preliminary results of a comparative effectiveness evaluation of adhesiolysis and caudal epidural injections in managing chronic low back pain secondary to spinal stenosis: A randomized, equivalence controlled trial. Pain Physician 2009; 12:E341-E354.

195. Manchikanti L, Singh V, Cash KA, Pampati V, Datta S. A comparative effectiveness evaluation of percutaneous adhesiolysis and epidural steroid injections in managing lumbar post surgery syndrome: A randomized, equivalence con- trolled trial. Pain Physician 2009; 12: E355-E368.

196. Manchikanti L, Cash KA, McManus CD, Pampati V, Smith HS. Preliminary results of randomized, equivalence trial of fluoroscopic caudal epidural injections in managing chronic low back pain: Part 1. Discogenic pain without disc herniation or radiculitis. Pain Physician 2008; 11:785-800.

197. Manchikanti L, Singh V, Cash KA, Pampati V, Damron KS, Boswell MV. Preliminary results of randomized, equivalence trial of fluoroscopic caudal epidural injections in managing chronic low back pain: Part 2. Disc herniation and radiculitis. Pain Physician 2008; 11:801-815.

198. Manchikanti L, Singh V, Cash KA, Pampati V, Datta S. Preliminary results of randomized, equivalence trial of fluoroscopic caudal epidural injections in managing chronic low back pain: Part 3. Post surgery syndrome. Pain Physician 2008; 11:817-831.

199. Manchikanti L, Cash KA, McManus CD, Pampati V, Abdi S. Preliminary results of randomized, equivalence trial of fluoroscopic caudal epidural injections in managing chronic low back pain: Part 4. Spinal stenosis. Pain Physician 2008; 11:833-848.

200. Manchikanti L, Cash KA, Pampati V, Wargo BW, Malla Y. Cervical epidural injections in chronic discogenic neck pain without disc herniation or radiculitis: Preliminary results of a randomized, double-blind, controlled trial. Pain Physician 2010; 13:E265-E278.

201. Manchikanti L, Cash KA, Pampati V, Wargo BW, Malla Y. The effectiveness of fluoroscopic cervical interlaminar epidural injections in managing chronic cervical disc herniation and radiculitis: Preliminary results of a randomized, doubleblind, controlled trial. Pain Physician 2010; 13:223-236.

202. Manchikanti L, Singh V, Falco FJE, Cash KA, Pampati V. Evaluation of the effectiveness of lumbar interlaminar epidural injections in managing chronic pain of lumbar disc herniation or radiculitis: A randomized, double-blind, controlled trial. Pain Physician 2010; 13:343-355.

203. Manchikanti L, Cash KA, McManus CD, Pampati V, Benyamin R. Preliminary results of a randomized, double-blind, controlled trial of fluoroscopic lumbar interlaminar epidural injections in managing chronic lumbar discogenic pain without disc herniation or radiculitis. Pain Physician 2010; 13:E279-E292. 
204. Manchikanti L, Singh V, Falco FJ, Cash KA, Fellows B. Cervical medial branch blocks for chronic cervical facet joint pain: A randomized double-blind, controlled trial with one-year follow-up. Spine (Phila Pa 1976) 2008; 33:18131820.

205. Manchikanti L, Singh V, Falco FJE, Cash KA, Pampati V, Fellows B. Comparative effectiveness of a one-year follow-up of thoracic medial branch blocks in management of chronic thoracic pain: A randomized, double-blind active controlled trial. Pain Physician 2010; 13:535-548.

206. Manchikanti L, Cash KA, McManus CD, Pampati V, Benyamin RM. A preliminary report of a randomized double-blind active controlled trial of fluoroscopic thoracic interlaminar epidural injections in managing chronic thoracic pain. Pain Physician 2010; 13:E357-E369.

207. Conn A, Buenaventura R, Datta S, Abdi $S$, Diwan S. Systematic review of caudal epidural injections in the management of chronic low back pain. Pain Physician 2009; 12:109-135.

208. Parr AT, Diwan S, Abdi S. Lumbar interlaminar epidural injections in managing chronic low back and lower extremity pain: A systematic review. Pain Physician 2009; 12:163-188.

209. Benyamin RM, Singh V, Parr AT, Conn A, Diwan S, Abdi S. Systematic review of the effectiveness of cervical epidurals in the management of chronic neck pain. Pain Physician 2009; 12:137-157.

210. Buenaventura RM, Datta $S$, Abdi $S$, Smith HS. Systematic review of therapeutic lumbar transforaminal epidural steroid injections. Pain Physician 2009; 12:233-251.

211. Epter RS, Helm S, Hayek SM, Benyamin RM, Smith HS, Abdi S. Systematic review of percutaneous adhesiolysis and management of chronic low back pain in post lumbar surgery syndrome. Pain Physician 2009; 12:361-378.

212. Hayek SM, Helm S, Benyamin RM, Singh $V$, Bryce DA, Smith HS. Effectiveness of spinal endoscopic adhesiolysis in post lumbar surgery syndrome: A systematic review. Pain Physician 2009; 12:419435.

213. Hirsch JA, Singh V, Falco FJE, Benyamin RM, Manchikanti L. Automated percutaneous lumbar discectomy for the contained herniated lumbar disc: A systematic assessment of evidence. Pain Physician 2009; 12:601-620.
214. Singh V, Manchikanti L, Benyamin RM, Helm S, Hirsch JA. Percutaneous lumbar laser disc decompression: A systematic review of current evidence. Pain Physician 2009; 12:573-588.

215. Singh V, Benyamin RM, Datta S, Falco FJE, Helm S, Manchikanti L. Systematic review of percutaneous lumbar mechanical disc decompression utilizing Dekompressor冈. Pain Physician 2009; 12:589-599.

216. Manchikanti L, Derby R, Benyamin RM, Helm S, Hirsch JA. A systematic review of mechanical lumbar disc decompression with nucleoplasty. Pain Physician 2009; 12:561-572.

217. Helm S, Hayek S, Benyamin RM, Manchikanti L. Systematic review of the effectiveness of thermal annular procedures in treating discogenic low back pain. Pain Physician 2009; 12:207-232.

218. Rupert MP, Lee $M$, Manchikanti L, Datta $S$, Cohen SP. Evaluation of sacroiliac joint interventions: A systematic appraisal of the literature. Pain Physician 2009; 12:399-418.

219. Atluri S, Datta S, Falco FJE, Lee M. Systematic review of diagnostic utility and therapeutic effectiveness of thoracic facet joint interventions. Pain Physician 2008; 11:611-629.

220. Falco FJE, Erhart S, Wargo BW, Bryce DA, Atluri S, Datta S, Hayek SM. Systematic review of diagnostic utility and therapeutic effectiveness of cervical facet joint interventions. Pain Physician 2009; 12:323-344.

221. Datta $S$, Lee $M$, Falco FJE, Bryce DA, Hayek SM. Systematic assessment of diagnostic accuracy and therapeutic utility of lumbar facet joint interventions. Pain Physician 2009; 12:437-460.

222. Frey ME, Manchikanti L, Benyamin RM, Schultz DM, Smith HS, Cohen SP. Spinal cord stimulation for patients with failed back surgery syndrome: A systematic review. Pain Physician 2009; 12:379397.

223. Patel VB, Manchikanti L, Singh V, Schultz DM, Hayek SM, Smith HS. Systematic review of intrathecal infusion systems for long-term management of chronic non-cancer pain. Pain Physician 2009; 12:345-360.

224. Nath S, Nath CA, Pettersson K. Percutaneous lumbar zygapophysial (facet) joint neurotomy using radiofrequency current, in the management of chronic low back pain: A randomized double- blind trial. Spine (Phila Pa 1976) 2008; 33:1291-1298.

225. Manchikanti L, Boswell MV, Rivera JJ, Pampati V, Damron KS, McManus CD, Brandon DE, Wilson SR. A randomized, controlled trial of spinal endoscopic adhesiolysis in chronic refractory low back and lower extremity pain. BMC Anesthesiol 2005; 5:10.

226. Manchikanti L, Rivera JJ, Pampati V, Damron KS, McManus CD, Brandon DE, Wilson SR. One day lumbar epidural adhesiolysis and hypertonic saline neurolysis in treatment of chronic low back pain: A randomized, double-blind trial. Pain Physician 2004; 7:177-186.

227. Pampati S, Cash KA, Manchikanti L. Accuracy of diagnostic lumbar facet joint nerve blocks: A 2-year follow-up of 152 patients diagnosed with controlled diagnostic blocks. Pain Physician 2009; 12:855-866.

228. Manchikanti L, Pampati S, Cash KA. Making sense of accuracy of diagnostic lumbar facet joint nerve blocks: An assessment of implications of 50\% relief, $80 \%$ relief, single block or controlled diagnostic blocks. Pain Physician 2010; 13:133-143.

229. Manchikanti L, Singh V, Cash KA, Pampati V, Datta $S$. Management of pain of post lumbar surgery syndrome: Oneyear results of a randomized, double blind controlled trial of fluoroscopic caudal epidural injections. Pain Physician 2010; 13:509-521.

230. Gerges FJ, Lipsitz SR, Nedeljkovic SS. A systematic review on the effectiveness of the nucleoplasty procedure for discogenic pain. Pain Physician 2010; 13:117132.

231. Graziotti P, Goucke R, for the Directors of the Australian Pain Society. The use of oral opioids in patients with chronic nonmalignant pain: Management strategies. Perth, Australia: Australian Pain Society; 2002. www.apsoc.org.au/pdfs/ opioid.pdf.

232. Chapman CR, Lipschitz DL, Angst MS, Chou R, Denisco RC, Donaldson GW, Fine PG, Foley KM, Gallagher RM, Gilson AM, Haddox JD, Horn SD, Inturrisi CE, Jick SS, Lipman AG, Loeser JD, Noble M, Porter L, Rowbotham MC, Schoelles KM, Turk DC, Volinn E, Von Korff MR, Webster LR, Weisner CM. Opioid pharmacotherapy for chronic non-cancer pain in the united states: a research guideline for developing an evidence-base. J Pain 2010; 11:807-829. 
